\title{
General Description of Nonnegative Waveforms up to Second Harmonic for Power Amplifier Modelling
}

\author{
Anamarija Juhas and Ladislav A. Novak \\ Department of Power, Electronics and Communication Engineering, Faculty of Technical Sciences, University of Novi Sad, \\ 21000 Novi Sad, Serbia \\ Correspondence should be addressed to Ladislav A. Novak; ladislav@uns.ac.rs
}

Received 9 April 2014; Accepted 21 May 2014; Published 25 June 2014

Academic Editor: Matjaz Perc

Copyright (C) 2014 A. Juhas and L. A. Novak. This is an open access article distributed under the Creative Commons Attribution License, which permits unrestricted use, distribution, and reproduction in any medium, provided the original work is properly cited.

\begin{abstract}
General description of nonnegative waveforms up to second harmonic in terms of independent (unconstrained) parameters is provided. Three important subclasses of the class of nonnegative waveforms are also fully characterised: nonnegative waveforms with maximal amplitude of fundamental harmonic for prescribed amplitude of second harmonic, nonnegative waveforms with maximal coefficient of cosine part of fundamental harmonic for prescribed coefficients of second harmonic, and nonnegative waveforms with at least one zero. We prove that members of the first two subclasses have at least one zero; that is, they also belong to the third subclass. Nonnegative cosine waveforms up to second harmonic are also considered and characterised. A number of case studies of practical interest for power amplifier (PA) design, involving nonnegative waveforms up to second harmonic, are also considered.
\end{abstract}

\section{Introduction}

In electrical engineering, the problem of shaping/modelling drain (collector, plate) waveforms in PA design, in order to improve efficiency, is of ultimate interest. It is largely related to the problem of finding nonnegative waveforms and as such attracted both engineers (e.g., see [1-7]) and mathematicians (e.g., see $[8,9]$ ).

The family of nonnegative waveforms up to second harmonic has proved to be of particular interest for range of modes named class-J or class- $\mathrm{B} / \mathrm{J}[6,10,11]$, inverse class F/J [12], external second harmonic injection [13], and PA with arbitrary harmonic terminations [4]. In spite of frequent usage of nonnegative waveforms up to second harmonic in PA design, according to our best knowledge, this is the first result providing general description of nonnegative waveforms up to second harmonic in terms of independent (unconstrained) parameters. This problem of finding general description which naturally arises from engineering practice has proved to be a nontrivial mathematical problem.
In this paper, we consider normalized waveform with first two harmonics (trigonometric polynomial of order two):

$$
T_{2}(\theta)=1+a_{1} \cos \theta+b_{1} \sin \theta+a_{2} \cos 2 \theta+b_{2} \sin 2 \theta,
$$

aiming to provide closed form expressions for coefficients $a_{1}$, $b_{1}, a_{2}$, and $b_{2}$ in terms of independent parameters such that the following condition holds:

$$
T_{2}(\theta) \geq 0 .
$$

Relations (1) and (2) refer to the class of nonnegative waveforms (Section 2).

We also consider a subclass of nonnegative waveforms that satisfy additional condition:

$$
T_{2}\left(\theta_{0}\right)=0,
$$

for at least one $\theta_{0}$ (Section 5). We prove that this subclass includes all nonnegative waveforms with maximal amplitude of fundamental harmonic, when second harmonic amplitude 


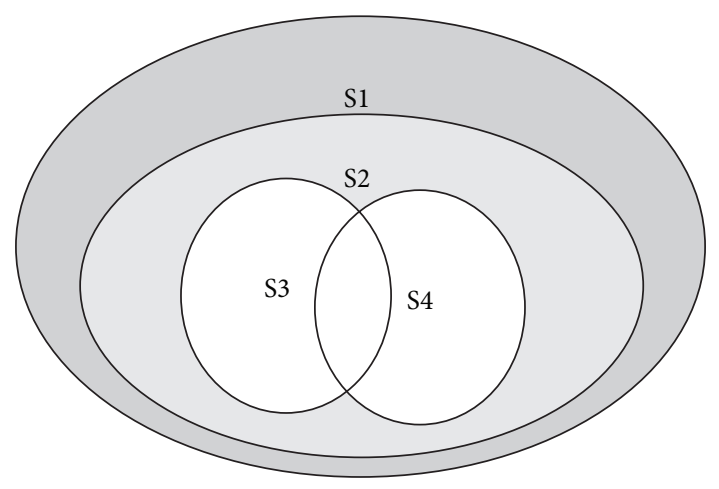

FIGURE 1: Class of nonnegative waveforms (S1) and its subclasses containing waveforms with at least one zero (S2), waveforms with maximal $a_{1}^{2}+b_{1}^{2}$ for prescribed $a_{2}^{2}+b_{2}^{2}$ (S3), and waveforms with $\left|a_{1}\right|_{\max }$ for prescribed $a_{2}$ and $b_{2}$ (S4).

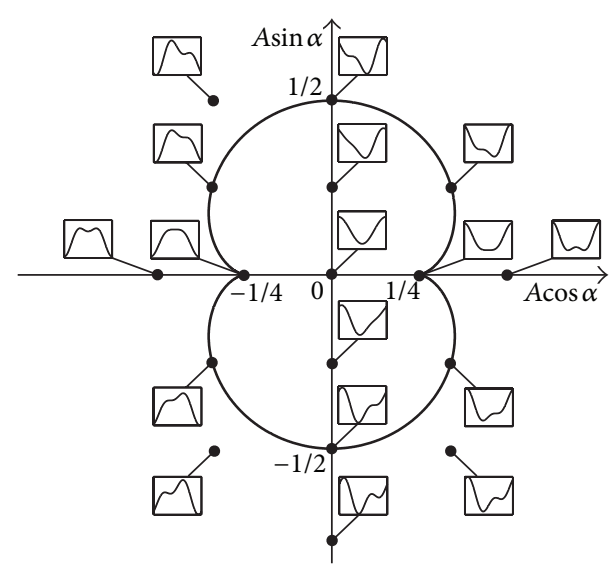

Figure 2: Parameter space $(A \cos \alpha, A \sin \alpha)$ and waveforms of the family (4).

is prescribed (Section 3), and all nonnegative waveforms with maximal absolute value of coefficient $a_{1}$, when coefficients $a_{2}$ and $b_{2}$ are prescribed (Section 4 ). General description of nonnegative cosine waveforms is provided in Section 6. In Section 7, a number of case studies related to the efficiency of PA with nonnegative waveforms up to second harmonic are considered. Figure 1 describes relationship between considered classes of nonnegative waveforms.

In the quest for shape zoology of waveforms of type (1), it is enough to consider the following generic two-parameter family of waveforms:

$$
f(\theta ; A, \alpha)=\cos \theta+A \cos (2 \theta+\alpha) .
$$

This is because all waveforms of type (1) can be obtained from (4) by appropriate usage of the following three operations: shifting along $\theta$ axis and introducing additive and/or multiplicative constants. Notice that these operations do not inherently change waveform shape.

To each waveform of type (4) corresponds a pair $(A, \alpha)$ or equivalently a pair $(A \cos \alpha, A \sin \alpha)$ and vice versa. The zoo of shapes is presented in Figure 2. Bolded curve in Figure 2 divides the parameter space $(A \cos \alpha, A \sin \alpha)$ into three disjoint subsets (inner part, outer part, and solid line itself) and helps in making classification of the shapes of waveforms. The points of this curve constitute the so-called catastrophe set and correspond to those pairs $(A, \alpha)$ for which there exist $\theta_{d}$ such that both first and second derivatives of the waveform $f(\theta ; A, \alpha)$ at $\theta_{d}$ are equal to zero:

$$
\begin{aligned}
& f^{(1)}\left(\theta_{d} ; A, \alpha\right)=0, \\
& f^{(2)}\left(\theta_{d} ; A, \alpha\right)=0 .
\end{aligned}
$$

An analogous consideration of waveform shapes with first and third harmonic is presented in [5].

Waveforms that correspond to the inner points have one minimum and one maximum, whereas the waveforms that correspond to the outer points have two minima and two maxima. Points on the solid line correspond to the waveforms with one minimum, one maximum, and one inflection point. Two cusp points $(A=1 / 4, \alpha=0)$ and $(A=1 / 4, \alpha=\pi)$ correspond to the maximally flat waveforms (e.g., see [5]), with maximally flat minimum and maximally flat maximum, respectively.

\section{General Description of Nonnegative Waveforms up to Second Harmonic}

In this section, we provide general description of nonnegative waveforms up to second harmonic in terms of four independent (unconstrained) parameters, along with a number of special cases.

The main result of this section is stated in the following proposition.

Proposition 1. For every waveform of type (1) satisfying condition (2), coefficients $a_{1}, b_{1}, a_{2}$, and $b_{2}$ can be expressed in terms of four independent (unconstrained) parameters $\xi, \psi$, $\varphi_{1}$, and $\varphi_{2}$ as

$$
\begin{aligned}
& a_{1}=\sin 2 \xi\left(\cos \psi \cos \varphi_{1}+\sin \psi \cos \varphi_{2}\right), \\
& b_{1}=\sin 2 \xi\left(\cos \psi \sin \varphi_{1}-\sin \psi \sin \varphi_{2}\right), \\
& a_{2}=\cos ^{2} \xi \sin 2 \psi \cos \left(\varphi_{1}-\varphi_{2}\right), \\
& b_{2}=\cos ^{2} \xi \sin 2 \psi \sin \left(\varphi_{1}-\varphi_{2}\right) .
\end{aligned}
$$

Proof. Let

$$
\underline{H}_{2}\left(e^{j \theta}\right)=c_{0}+j d_{0}+\left(c_{1}+j d_{1}\right) e^{j \theta}+\left(c_{2}+j d_{2}\right) e^{2 j \theta}
$$

be a complex polynomial on the unit circle $(j=\sqrt{-1})$ such that parameters $c_{0}, c_{1}, c_{2}, d_{0}, d_{1}$, and $d_{2}$ satisfy the following constraint:

$$
1=c_{0}^{2}+c_{1}^{2}+c_{2}^{2}+d_{0}^{2}+d_{1}^{2}+d_{2}^{2} .
$$

According to the classical result related to trigonometric polynomials ([8]; see also [14]), all trigonometric polynomials of type (1) satisfying condition (2) can be expressed as

$$
T_{2}(\theta)=\operatorname{Re}^{2}\left\{\underline{H}_{2}\left(e^{j \theta}\right)\right\}+\operatorname{Im}^{2}\left\{\underline{H}_{2}\left(e^{j \theta}\right)\right\} .
$$


As shown in [8], coefficients $a_{1}, b_{1}, a_{2}$, and $b_{2}$ can be expressed in terms of six parameters $c_{0}, c_{1}, c_{2}, d_{0}, d_{1}$, and $d_{2}$ in the following form:

$$
\begin{aligned}
& a_{1}=2\left(c_{0} c_{1}+c_{1} c_{2}+d_{0} d_{1}+d_{1} d_{2}\right), \\
& a_{2}=2\left(c_{0} c_{2}+d_{0} d_{2}\right), \\
& b_{1}=2\left(d_{0} c_{1}+d_{1} c_{2}-c_{0} d_{1}-c_{1} d_{2}\right), \\
& b_{2}=2\left(c_{2} d_{0}-c_{0} d_{2}\right),
\end{aligned}
$$

providing that relation (11) holds.

In what follows, we will show that coefficients $a_{1}, b_{1}, a_{2}$, and $b_{2}$ can be expressed in terms of four arbitrary parameters, instead of six parameters $c_{0}, c_{1}, c_{2}, d_{0}, d_{1}$, and $d_{2}$ constrained by (11).

We start with the observation that hypersphere (11) can be written in terms of new parameter $\xi$ as

$$
\begin{gathered}
c_{1}^{2}+d_{1}^{2}=\sin ^{2} \xi, \\
c_{0}^{2}+c_{2}^{2}+d_{0}^{2}+d_{2}^{2}=\cos ^{2} \xi .
\end{gathered}
$$

Furthermore, the sphere (17) can be also parameterised by introducing new parameter $\vartheta$ as follows:

$$
\begin{aligned}
& c_{1}=\sin \xi \cos \vartheta \\
& d_{1}=\sin \xi \sin \vartheta .
\end{aligned}
$$

In a similar way, hypersphere (18) can be parameterised by introducing further three parameters $\psi, \phi_{1}$, and $\phi_{2}$ as follows:

$$
\begin{aligned}
& c_{0}=\cos \xi \cos \psi \cos \phi_{1}, \\
& c_{2}=\cos \xi \sin \psi \cos \phi_{2}, \\
& d_{0}=\cos \xi \cos \psi \sin \phi_{1}, \\
& d_{2}=\cos \xi \sin \psi \sin \phi_{2} .
\end{aligned}
$$

Inserting (21)-(24) into (13)-(16) yields

$$
\begin{aligned}
& a_{1}=\sin 2 \xi\left(\cos \psi \cos \left(\phi_{1}-\vartheta\right)+\sin \psi \cos \left(\phi_{2}-\vartheta\right)\right), \\
& b_{1}=\sin 2 \xi\left(\cos \psi \sin \left(\phi_{1}-\vartheta\right)-\sin \psi \sin \left(\phi_{2}-\vartheta\right)\right) \\
& a_{2}=\cos ^{2} \xi \sin 2 \psi \cos \left(\phi_{1}-\phi_{2}\right) \\
& b_{2}=\cos ^{2} \xi \sin 2 \psi \sin \left(\phi_{1}-\phi_{2}\right) .
\end{aligned}
$$

By introducing parameters $\varphi_{1}$ and $\varphi_{2}$ defined as

$$
\begin{aligned}
& \varphi_{1}=\phi_{1}-\vartheta \\
& \varphi_{2}=\phi_{2}-\vartheta
\end{aligned}
$$

coefficients $a_{1}, b_{1}, a_{2}$, and $b_{2}$ (see (25)-(28)) can be expressed in four parameters only $\xi, \psi, \varphi_{1}$ and $\varphi_{2}$, taking into consideration that

$$
\phi_{1}-\phi_{2}=\varphi_{1}-\varphi_{2}
$$

which completes the proof.
Notice that substitution of (6)-(9) into (1) leads to the following forms of nonnegative waveforms up to second harmonic:

$$
\begin{aligned}
T_{2}(\theta)= & +\sin 2 \xi\left(\cos \psi \cos \left(\theta-\varphi_{1}\right)\right. \\
& \left.+\sin \psi \cos \left(\theta+\varphi_{2}\right)\right) \\
& +\cos ^{2} \xi \sin 2 \psi \cos \left(2 \theta-\varphi_{1}+\varphi_{2}\right) \\
T_{2}(\theta)=\left[\left(\cos \psi \cos \left(\theta-\varphi_{1}\right)\right.\right. & \left.\left.+\sin \psi \cos \left(\theta+\varphi_{2}\right)\right) \cos \xi+\sin \xi\right]^{2} \\
& +\left(\cos \psi \sin \left(\theta-\varphi_{1}\right)-\sin \psi \sin \left(\theta+\varphi_{2}\right)\right)^{2} \cos ^{2} \xi
\end{aligned}
$$

Remark 2. Maximum value of amplitude of fundamental harmonic of nonnegative waveform of type (1) is $\sqrt{2}$ and corresponding amplitude of second harmonic is $1 / 2$.

In order to show that, let

$$
\begin{aligned}
& A_{2}=\cos ^{2} \xi \sin 2 \psi, \\
& A_{1}^{2}=\sin ^{2} 2 \xi\left(1+\sin 2 \psi \cos \left(\varphi_{1}+\varphi_{2}\right)\right) .
\end{aligned}
$$

From (8)-(9) and (6)-(7), it follows that

$$
\begin{aligned}
& a_{2}^{2}+b_{2}^{2}=\cos ^{4} \xi \sin ^{2} 2 \psi \\
& a_{1}^{2}+b_{1}^{2}=\sin ^{2} 2 \xi\left(1+\sin 2 \psi \cos \left(\varphi_{1}+\varphi_{2}\right)\right),
\end{aligned}
$$

respectively. According to (32) and (34), amplitude of second harmonic of nonnegative waveform of type (1) is

$$
\left|A_{2}\right|=\sqrt{a_{2}^{2}+b_{2}^{2}} .
$$

According to (33) and (35), amplitude of fundamental harmonic of nonnegative waveform of type (1) is

$$
\left|A_{1}\right|=\sqrt{a_{1}^{2}+b_{1}^{2}} .
$$

It is easy to see that, according to (33), the maximum value $A_{1}^{2}=2$ can be attained if and only if $\sin ^{2} 2 \xi=1$ and $\sin 2 \psi \cos \left(\varphi_{1}+\varphi_{2}\right)=1$. Therefore, maximum value of amplitude of fundamental harmonic $\left|A_{1}\right|$ of nonnegative waveform of type (1) is $\sqrt{2}$. From $\sin ^{2} 2 \xi=1$ and $\sin 2 \psi \cos \left(\varphi_{1}+\varphi_{2}\right)=1$, it also follows that $\cos ^{2} \xi=1 / 2$ and $\sin ^{2} 2 \psi=1$. Consequently, (34) and (36) imply that amplitude of second harmonic $\left|A_{2}\right|$ of nonnegative waveform of type (1) with $\left|A_{1}\right|=\sqrt{2}$ is $1 / 2$.

Remark 3. A number of special cases of nonnegative waveforms (1) with $a_{2}=0$ or $b_{2}=0$, each corresponding to a specific parameter choice, are listed in Tables 1, 2, 3, 4, 5, 6, and 7.

\section{Nonnegative Waveforms with Maximal Fundamental Harmonic Amplitude}

In this section, we provide general description of nonnegative waveforms of type (1) with maximal amplitude of fundamental harmonic for prescribed second harmonic amplitude $\left|A_{2}\right|$. 
TABLE 1: Coefficients of nonnegative waveforms with $a_{2}=0$.

\begin{tabular}{lc}
\hline Constraint & $a_{2}=0$ \\
Condition & $\cos \left(\varphi_{1}-\varphi_{2}\right)=0$ \\
Parameter choice & $\varphi_{2}=\varphi_{1}-\pi / 2$ \\
& $a_{1}=\sin 2 \xi \cos \left(\psi-\varphi_{1}\right)$ \\
Coefficients & $b_{1}=\sin 2 \xi \sin \left(\psi+\varphi_{1}\right)$ \\
& $b_{2}=\cos ^{2} \xi \sin 2 \psi$ \\
\hline
\end{tabular}

TABLE 2: Coefficients of nonnegative waveforms with $a_{1}=a_{2}=0$.

\begin{tabular}{lc}
\hline Constraint & $a_{1}=a_{2}=0$ \\
Condition & $\cos \left(\varphi_{1}-\varphi_{2}\right)=0$ \\
& $\cos \left(\psi-\varphi_{1}\right)=0$ \\
Parameter choice & $\varphi_{1}=\psi+\pi / 2, \varphi_{2}=\psi$ \\
Coefficients & $b_{1}=\sin 2 \xi \cos 2 \psi$ \\
& $b_{2}=\cos ^{2} \xi \sin 2 \psi$ \\
\hline
\end{tabular}

TABLE 3: Coefficients of nonnegative waveforms with $b_{1}=a_{2}=0$.

\begin{tabular}{lc}
\hline Constraint & $b_{1}=a_{2}=0$ \\
Condition & $\cos \left(\varphi_{1}-\varphi_{2}\right)=0$ \\
& $\sin \left(\psi+\varphi_{1}\right)=0$ \\
Parameter choice & $\varphi_{1}=-\psi, \varphi_{2}=-\psi-\pi / 2$ \\
Coefficients & $a_{1}=\sin 2 \xi \cos 2 \psi$ \\
& $b_{2}=\cos ^{2} \xi \sin 2 \psi$ \\
\hline
\end{tabular}

TABLE 4: Coefficients of nonnegative waveforms with $a_{2}=b_{2}=0$.

\begin{tabular}{lc}
\hline Constraint & $a_{2}=b_{2}=0$ \\
Condition & $\sin 2 \psi=0$ \\
Parameter choice & $\psi=0$ \\
Coefficients & $a_{1}=\sin 2 \xi \cos \varphi_{1}$ \\
& $b_{1}=\sin 2 \xi \sin \varphi_{1}$ \\
\hline
\end{tabular}

TABLE 5: Coefficients of nonnegative waveforms with $b_{2}=0$.

\begin{tabular}{lc}
\hline Constraint & $b_{2}=0$ \\
Condition & $\sin \left(\varphi_{1}-\varphi_{2}\right)=0$ \\
Parameter choice & $\varphi_{2}=\varphi_{1}$ \\
& $a_{1}=\sin 2 \xi \cos \varphi_{1}(\cos \psi+\sin \psi)$ \\
Coefficients & $b_{1}=\sin 2 \xi \sin \varphi_{1}(\cos \psi-\sin \psi)$ \\
& $a_{2}=\cos ^{2} \xi \sin 2 \psi$ \\
\hline
\end{tabular}

TABLE 6: Coefficients of nonnegative waveforms with $b_{1}=b_{2}=0$.

\begin{tabular}{lc}
\hline Constraint & $b_{1}=b_{2}=0$ \\
Condition & $\sin \varphi_{1}=\sin \varphi_{2}=0$ \\
Parameter choice & $\varphi_{2}=\varphi_{1}=0$ \\
Coefficients & $a_{1}=\sin 2 \xi(\cos \psi+\sin \psi)$ \\
& $a_{2}=\cos ^{2} \xi \sin 2 \psi$ \\
\hline
\end{tabular}

TABLE 7: Coefficients of nonnegative waveforms with $a_{1}=b_{2}=0$.

\begin{tabular}{lc}
\hline Constraint & $a_{1}=b_{2}=0$ \\
Condition & $\cos \varphi_{1}=\cos \varphi_{2}=0$ \\
Parameter choice & $\varphi_{2}=\varphi_{1}=\pi / 2$ \\
Coefficients & $b_{1}=\sin 2 \xi(\cos \psi-\sin \psi)$ \\
& $a_{2}=\cos ^{2} \xi \sin 2 \psi$ \\
\hline
\end{tabular}

According to (34) and (36), for nonnegative waveforms of type (1), the following relation holds:

$$
0 \leq\left|A_{2}\right| \leq 1
$$

This explains why amplitude of second harmonic $\left|A_{2}\right|$ in Proposition 4 goes through interval $[0,1]$ only.

Proposition 4. Every nonnegative waveform of type (1) with maximal amplitude of fundamental harmonic for prescribed amplitude $\left|A_{2}\right|$ of second harmonic can be represented as

$$
\begin{aligned}
& T_{2}(\theta)=\left[1-\cos \left(\theta-\theta_{0}\right)\right]\left[1-\left|A_{2}\right|-2\left|A_{2}\right| \cos \left(\theta-\theta_{0}\right)\right], \\
& \text { if } 0 \leq\left|A_{2}\right| \leq 1 / 3 \text {, or } \\
& T_{2}(\theta)=2\left|A_{2}\right|\left[1-\cos \left(\theta-\theta_{0}\right)\right]\left[1-\cos \left(\theta-\theta_{0}^{\prime}\right)\right],
\end{aligned}
$$

if $1 / 3 \leq\left|A_{2}\right| \leq 1$, providing that

$$
\cos \left(\theta_{0}-\theta_{0}^{\prime}\right)=-2+\frac{1}{\left|A_{2}\right|}
$$

Remark 5. As an immediate consequence of (39) and (40), it follows that every nonnegative waveform of type (1) with maximal amplitude of fundamental harmonic for prescribed amplitude of second harmonic has at least one zero.

Remark 6. Conversion of (39) and (40) into an additive form and comparison with (1) immediately leads to the explicit form of coefficients of nonnegative waveforms of type (1) with maximal amplitude of fundamental harmonic for prescribed second harmonic amplitude.

Conversion of (39) into an additive form lead to the following explicit form of coefficients:

$$
\begin{aligned}
& a_{1}=-\left(1+\left|A_{2}\right|\right) \cos \theta_{0}, \\
& a_{2}=\left|A_{2}\right| \cos 2 \theta_{0}, \\
& b_{1}=-\left(1+\left|A_{2}\right|\right) \sin \theta_{0}, \\
& b_{2}=\left|A_{2}\right| \sin 2 \theta_{0} .
\end{aligned}
$$

From (39), it follows immediately that $T_{2}(\theta)$ is nonnegative if and only if $0 \leq\left|A_{2}\right| \leq 1 / 3$. 


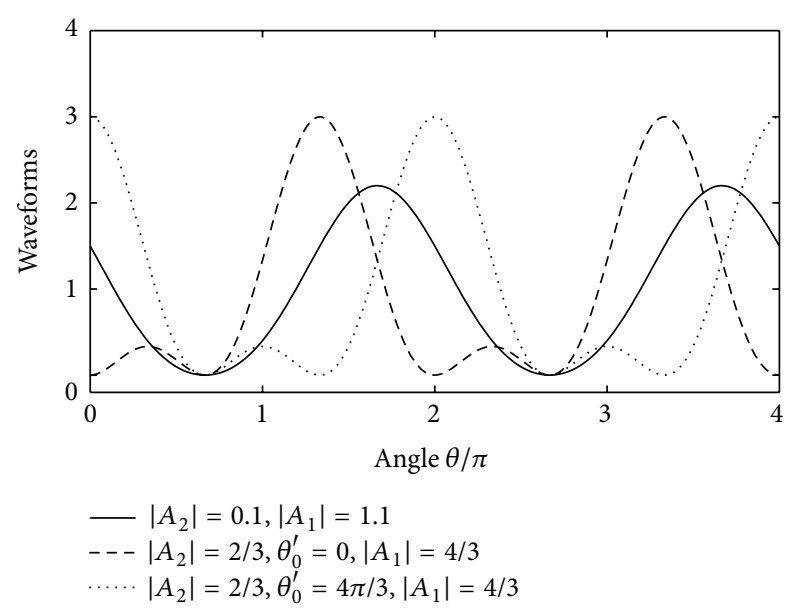

FIGURE 3: Nonnegative waveforms with maximal amplitude of fundamental harmonic for prescribed second harmonic amplitude and $\theta_{0}=2 \pi / 3$.

Conversion of (40) into an additive form lead to the following explicit form of coefficients:

$$
\begin{aligned}
& a_{1}=-2\left|A_{2}\right|\left(\cos \theta_{0}+\cos \theta_{0}^{\prime}\right), \\
& a_{2}=\left|A_{2}\right| \cos \left(\theta_{0}+\theta_{0}^{\prime}\right), \\
& b_{1}=-2\left|A_{2}\right|\left(\sin \theta_{0}+\sin \theta_{0}^{\prime}\right), \\
& b_{2}=\left|A_{2}\right| \sin \left(\theta_{0}+\theta_{0}^{\prime}\right) .
\end{aligned}
$$

It is easy to see that $\cos \left(\theta_{0}-\theta_{0}^{\prime}\right)=-2+1 /\left|A_{2}\right|$ implies $1 / 3 \leq$ $\left|A_{2}\right| \leq 1$ and $\cos \left(\theta_{0}-\theta_{0}^{\prime}\right)=1$ if and only if $\left|A_{2}\right|=1 / 3$.

Three examples of nonnegative waveforms with maximal amplitude of fundamental harmonic for prescribed second harmonic amplitude and prescribed position of zero $\theta_{0}=$ $2 \pi / 3$, are presented in Figure 3 for $\left|A_{2}\right|=0.1$ and $\left|A_{2}\right|=$ $2 / 3$. The waveform represented by solid line corresponds to $\left|A_{2}\right|=0.1$ and the other two waveforms correspond to $\left|A_{2}\right|=2 / 3$ (notice that $\left|A_{2}\right|=2 / 3$, according to (41), implies $\left.\theta_{0}^{\prime}=\theta_{0} \pm 2 \pi / 3\right)$.

Remark 7. Nonnegative waveforms with maximal amplitude of fundamental harmonic when $\left|A_{2}\right|=1 / 3$ can be expressed in the form

$$
T_{2}(\theta)=\frac{2}{3}\left[1-\cos \left(\theta-\theta_{0}\right)\right]^{2} .
$$

Waveforms (44) are so-called maximally flat waveform of type (1) (e.g., see [5]).

Remark 8. According to (42), (43), and (41), maximal amplitude of fundamental harmonic of nonnegative waveforms of type (1) can be expressed as a function of second harmonic amplitude (see Figure 4):

$$
\left|A_{1}\right|= \begin{cases}1+\left|A_{2}\right| & \text { for } 0 \leq\left|A_{2}\right| \leq \frac{1}{3}, \\ \sqrt{8\left|A_{2}\right|\left(1-\left|A_{2}\right|\right)} & \text { for } \frac{1}{3} \leq\left|A_{2}\right| \leq 1 .\end{cases}
$$

Proof of Proposition 4. Let us consider nonnegative waveform $T_{2}(\theta)$ of type (1) and let $\theta_{0}$ be a position of its global minimum; that is, $0 \leq T_{2}\left(\theta_{0}\right) \leq T_{2}(\theta)$.

If $A_{2}=0$, then, according to (32), $\cos \xi=0$ and/or $\sin 2 \psi=0$. For $\cos \xi=0$ from (33) it follows that $\left|A_{1}\right|=0$. On the other hand, $\sin 2 \psi=0$, according to (33), implies $\left|A_{1}\right|=|\sin 2 \xi|$. Therefore, maximal amplitude of fundamental harmonic is equal to 1 and hence $T_{2}(\theta)=$ $1-\cos \left(\theta-\theta_{0}\right)$, which coincides with (39) for $\left|A_{2}\right|=0$.

In what follows, we assume that $\left|A_{2}\right| \neq 0$, which, from (32), implies $\sin 2 \psi \neq 0$. Since $A_{2}$ does not depend on $\varphi_{1}$ and $\varphi_{2}$, according to (33), the maximal amplitude of the fundamental harmonic can be attained if and only if

$$
\sin 2 \psi \cos \left(\varphi_{1}+\varphi_{2}\right)=|\sin 2 \psi|
$$

Relation (46) and $\sin 2 \psi \neq 0$ imply $\left|\cos \left(\varphi_{1}+\varphi_{2}\right)\right|=1$. Therefore, $\cos \varphi_{2}=\cos \varphi_{1} \cos \left(\varphi_{1}+\varphi_{2}\right)$ and $\sin \varphi_{2}=$ $-\sin \varphi_{1} \cos \left(\varphi_{1}+\varphi_{2}\right)$ hold, which further, from (6) and (7), imply

$$
\begin{aligned}
& a_{1}=A_{1} \cos \varphi_{1}, \\
& b_{1}=A_{1} \sin \varphi_{1},
\end{aligned}
$$

providing that

$$
A_{1}=\sin 2 \xi\left(\cos \psi+\sin \psi \cos \left(\varphi_{1}+\varphi_{2}\right)\right) .
$$

Similarly, (46) implies sin $2 \psi \cos \left(\varphi_{1}-\varphi_{2}\right)=|\sin 2 \psi| \cos 2 \varphi_{1}$ and $\sin 2 \psi \sin \left(\varphi_{1}-\varphi_{2}\right)=|\sin 2 \psi| \sin 2 \varphi_{1}$, which further, according to (8), (9), and (32), imply

$$
\begin{aligned}
& a_{2}=\left|A_{2}\right| \cos 2 \varphi_{1}, \\
& b_{2}=\left|A_{2}\right| \sin 2 \varphi_{1} .
\end{aligned}
$$

Insertion of (47) and (49) into (1) lead to $T_{2}(\theta)=1+$ $A_{1} \cos \left(\theta-\varphi_{1}\right)+\left|A_{2}\right| \cos \left(2 \theta-2 \varphi_{1}\right)$, which can be rewritten as

$$
\begin{aligned}
T_{2}(\theta)= & \frac{8\left|A_{2}\right|\left(1-\left|A_{2}\right|\right)-A_{1}^{2}}{8\left|A_{2}\right|} \\
& +\frac{\left[A_{1}+4\left|A_{2}\right| \cos \left(\theta-\varphi_{1}\right)\right]^{2}}{8\left|A_{2}\right|} .
\end{aligned}
$$

Let $\theta_{0}$ be a position of global minimum of $T_{2}(\theta)$; that is, $0 \leq$ $T_{2}\left(\theta_{0}\right) \leq T_{2}(\theta)$. It is easy to see that $T_{2}(\theta)$ is minimal when $\left[A_{1}+4\left|A_{2}\right| \cos \left(\theta-\varphi_{1}\right)\right]^{2}$ is minimal. In what follows, we will consider two cases: Case (i) $\left|A_{1}\right| \geq 4\left|A_{2}\right|$ and Case (ii) $\left|A_{1}\right|<$ $4\left|A_{2}\right|$.

In Case (i), there are two options for expression $\left[A_{1}+4\left|A_{2}\right| \cos \left(\theta-\varphi_{1}\right)\right]^{2}$ to be minimal: either for $\cos \left(\theta_{0}-\right.$ $\left.\varphi_{1}\right)=1$ and $A_{1}<0$ or for $\cos \left(\theta_{0}-\varphi_{1}\right)=-1$ and $A_{1}>0$. Furthermore, $T_{2}\left(\theta_{0}\right) \geq 0$ implies $8\left|A_{2}\right|\left(1-\left|A_{2}\right|\right)-A_{1}^{2}+\left(\left|A_{1}\right|-\right.$ $\left.4\left|A_{2}\right|\right)^{2} \geq 0$, which is equivalent to $\left|A_{1}\right| \leq 1+\left|A_{2}\right|$. Therefore, maximal amplitude of fundamental harmonic is

$$
\left|A_{1}\right|=1+\left|A_{2}\right| \text {. }
$$




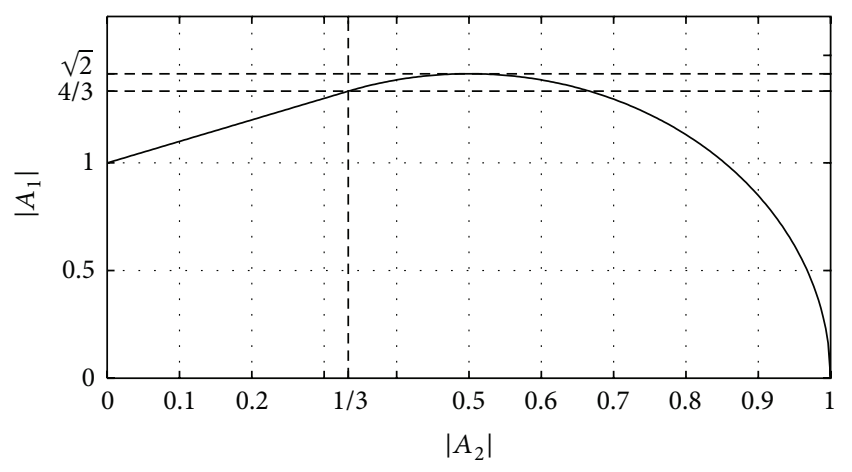

FIGURE 4: Maximal amplitude of fundamental harmonic as a function of $\left|A_{2}\right|$.

From $\left|A_{1}\right| \geq 4\left|A_{2}\right|$ and (51), it follows that $1+\left|A_{2}\right| \geq 4\left|A_{2}\right|$ and therefore

$$
0<\left|A_{2}\right| \leq \frac{1}{3} .
$$

The first option of the Case (i) reduces to $\theta_{0}=\varphi_{1}$ and $A_{1}=$ $-1-\left|A_{2}\right|$, while the second option of the Case (i) reduces to $\theta_{0}=\varphi_{1}+\pi$ and $A_{1}=1+\left|A_{2}\right|$. Substitution of any of these options into (50) results in

$$
T_{2}(\theta)=\frac{\left[1+\left|A_{2}\right|-4\left|A_{2}\right| \cos \left(\theta-\theta_{0}\right)\right]^{2}}{8\left|A_{2}\right|}-\frac{\left(3\left|A_{2}\right|-1\right)^{2}}{8\left|A_{2}\right|} .
$$

Factorisation of (53) immediately lead to (39), which proves that (39) holds for $0<\left|A_{2}\right| \leq 1 / 3$.

From the previous consideration, it follows that, in Case (ii), it is enough to consider

$$
\frac{1}{3}<\left|A_{2}\right| \leq 1
$$

only. In this case, according to (50), position of global minimum $\theta_{0}$ corresponds to the situation when $A_{1}+4\left|A_{2}\right| \cos \left(\theta_{0}-\right.$ $\left.\varphi_{1}\right)=0$ holds. Consequently,

$$
A_{1}=-4\left|A_{2}\right| \cos \left(\theta_{0}-\varphi_{1}\right) .
$$

Also, $T_{2}\left(\theta_{0}\right) \geq 0$ implies $8\left|A_{2}\right|\left(1-\left|A_{2}\right|\right)-A_{1}^{2} \geq 0$ and therefore maximal amplitude of fundamental harmonic is

$$
\left|A_{1}\right|=\sqrt{8\left|A_{2}\right|\left(1-\left|A_{2}\right|\right)} .
$$

Substitution of (55) and (56) into (50) lead to $T_{2}(\theta)=$ $2\left|A_{2}\right|\left[\cos \left(\theta-\varphi_{1}\right)-\cos \left(\theta_{0}-\varphi_{1}\right)\right]^{2}$, which can be rewritten as

$$
T_{2}(\theta)=2\left|A_{2}\right|\left[1-\cos \left(\theta-\theta_{0}\right)\right]\left[1-\cos \left(\theta+\theta_{0}-2 \varphi_{1}\right)\right] .
$$

Also, from (55) and (56), it follows that

$$
\cos \left(2 \theta_{0}-2 \varphi_{1}\right)=-2+\frac{1}{\left|A_{2}\right|}
$$

Substituting $2 \varphi_{1}-\theta_{0}$ in (57)-(58) by $\theta_{0}^{\prime}$ lead to (40)-(41), which proves that (40)-(41) hold for $1 / 3<\left|A_{2}\right| \leq 1$.

Finally, for $\left|A_{2}\right|=1 / 3$, from (41), it follows that $\cos \left(\theta_{0}-\right.$ $\left.\theta_{0}^{\prime}\right)=1$, which further implies $\cos \left(\theta-\theta_{0}^{\prime}\right)=\cos \left(\theta-\theta_{0}\right)$ and therefore (40) and (39) coincide for $A_{2}=1 / 3$. This completes the proof.

\section{Nonnegative Waveforms with Maximal Coefficient of Cosine Part of Fundamental Harmonic}

Problem of finding nonnegative waveforms with maximal absolute value of coefficient $a_{1}$ is of particular interest in PA efficiency analysis. In a number of cases of interest, one waveform of the voltage-current pair (e.g., current) is already known and usually has only cosine part of fundamental harmonic (e.g., see [7]). In such cases, the problem of finding maximal efficiency for prescribed second harmonic impedance can be reduced to the problem of finding nonnegative voltage waveform with maximal absolute value of coefficient $a_{1}$ providing that coefficients $a_{2}$ and $b_{2}$ are prescribed (see also Section 7.3).

The following proposition provides closed form expressions for nonnegative waveforms with maximal absolute value of coefficient $a_{1}$ for prescribed second harmonic coefficients $a_{2}$ and $b_{2}$.

Proposition 9. Every nonnegative waveform of type (1) with maximal absolute value of coefficient $a_{1}$ for prescribed second harmonic coefficients $a_{2}$ and $b_{2}$ can be represented as

$$
T_{2}(\theta)=[1-\cos \theta]\left[1-a_{2}-2 a_{2} \cos \theta-2 b_{2} \sin \theta\right],
$$

if $2\left|A_{2}\right| \leq 1-a_{2}$ and $a_{1} \leq 0$,

$$
T_{2}(\theta)=[1+\cos \theta]\left[1-a_{2}+2 a_{2} \cos \theta+2 b_{2} \sin \theta\right],
$$

if $2\left|A_{2}\right| \leq 1-a_{2}$ and $a_{1} \geq 0$, or

$$
T_{2}(\theta)=2\left|A_{2}\right|\left[1-\cos \left(\theta-\theta_{0}\right)\right]\left[1-\cos \left(\theta-\theta_{0}^{\prime}\right)\right],
$$

if $2\left|A_{2}\right| \geq 1-a_{2}$, providing that

$$
\begin{aligned}
& \cos \frac{\theta_{0}-\theta_{0}^{\prime}}{2}=-\operatorname{sgn}\left(a_{1}\right) \sqrt{\frac{1-\left|A_{2}\right|}{2\left|A_{2}\right|}}, \\
& \cos \frac{\theta_{0}+\theta_{0}^{\prime}}{2}=\sqrt{\frac{\left|A_{2}\right|+a_{2}}{2\left|A_{2}\right|}}, \\
& \sin \frac{\theta_{0}+\theta_{0}^{\prime}}{2}=\operatorname{sgn}\left(b_{2}\right) \sqrt{\frac{\left|A_{2}\right|-a_{2}}{2\left|A_{2}\right|}} .
\end{aligned}
$$

Remark 10. As an immediate consequence of (59)-(61), it follows that every nonnegative waveform of type (1) with maximal absolute value of coefficient $a_{1}$, for prescribed second harmonic coefficients $a_{2}$ and $b_{2}$, has at least one zero.

Remark 11. Conversion of (59)-(61) into an additive form and comparison with (1) immediately lead to the explicit form of 
coefficients $a_{1}$ and $b_{1}$. For $2\left|A_{2}\right| \leq 1-a_{2}$, conversion of (59) and (60) into an additive form lead to

$$
\begin{array}{cc}
a_{1}=-\left(1+a_{2}\right), & b_{1}=-2 b_{2}, \\
a_{1}=\left(1+a_{2}\right), & b_{1}=2 b_{2},
\end{array}
$$

respectively. Notice that $\left|a_{2}\right| \leq\left|A_{2}\right| \leq 1 / 3$ implies

$$
\begin{aligned}
& {\left[\left(1+a_{2}\right)^{2}+4 b_{2}^{2}\right]-\left[1+\left|A_{2}\right|\right]^{2}} \\
& \quad=\left(\left|A_{2}\right|-a_{2}\right)\left(3\left|A_{2}\right|+3 a_{2}-2\right) \leq 0 .
\end{aligned}
$$

On the other hand, $1 / 3<\left|A_{2}\right| \leq\left(1-a_{2}\right) / 2$ implies $1+3 a_{2}-$ $6\left|A_{2}\right|<4\left(1-3\left|A_{2}\right|\right)<0$ and therefore

$$
\begin{aligned}
& {\left[\left(1+a_{2}\right)^{2}+4 b_{2}^{2}\right]-\left[8\left|A_{2}\right|\left(1-\left|A_{2}\right|\right)\right]} \\
& \quad=\left(1-a_{2}-2\left|A_{2}\right|\right)\left(1+3 a_{2}-6\left|A_{2}\right|\right) \leq 0 .
\end{aligned}
$$

According to (63) and (64), fundamental harmonic amplitude of waveforms (59)-(60) is $\sqrt{\left(1+a_{2}\right)^{2}+4 b_{2}^{2}}$. Its value, according to (65)-(66), is less than maximal value of fundamental harmonic amplitude for prescribed second harmonic amplitude (see (45)), except for $\left|A_{2}\right|=a_{2} \leq 1 / 3$ or $2\left|A_{2}\right|=$ $1-a_{2}$ when they are equal.

For $2\left|A_{2}\right| \geq 1-a_{2}$, conversion of (61) into an additive form together with (62) lead to

$$
\begin{aligned}
& a_{1}=-2 \sqrt{1-\left|A_{2}\right|} \sqrt{\left|A_{2}\right|+a_{2}}, \\
& b_{1}=-\operatorname{sgn}\left(b_{2}\right) 2 \sqrt{1-\left|A_{2}\right|} \sqrt{\left|A_{2}\right|-a_{2}},
\end{aligned}
$$

if $a_{1} \leq 0$, or

$$
\begin{aligned}
& a_{1}=2 \sqrt{1-\left|A_{2}\right|} \sqrt{\left|A_{2}\right|+a_{2}}, \\
& b_{1}=\operatorname{sgn}\left(b_{2}\right) 2 \sqrt{1-\left|A_{2}\right|} \sqrt{\left|A_{2}\right|-a_{2},}
\end{aligned}
$$

if $a_{1} \geq 0$. According to (67) and (68), amplitude of fundamental harmonic of waveform (61) is $\sqrt{8\left|A_{2}\right|\left(1-\left|A_{2}\right|\right)}$ and according to (45) (notice that $2\left|A_{2}\right| \geq 1-a_{2}$ implies $\left|A_{2}\right| \geq$ $1 / 3)$ it coincides with maximal amplitude of fundamental harmonic for prescribed amplitude of second harmonic.

The contours of maximal absolute value of coefficient $a_{1}$ as a function of coefficients $a_{2}$ and $b_{2}$ are plotted in Figure 5 .

Two examples of nonnegative waveforms with the same maximal absolute value of coefficient $a_{1}$ (one with $a_{1}<$ 0 and the other with $a_{1}>0$ ) for prescribed coefficients $a_{2}=0.1$ and $b_{2}=0.3$ (corresponding to the case $2\left|A_{2}\right| \leq$ $1-a_{2}$ ) are presented in Figure 6. Another two examples of nonnegative waveforms with the same maximal absolute value of coefficient $a_{1}$ (one with $a_{1}<0$ and the other with $\left.a_{1}>0\right)$ for prescribed coefficients $a_{2}=0.5$ and $b_{2}=0.3$ (corresponding to the case $2\left|A_{2}\right| \geq 1-a_{2}$ ) are presented in Figure 7.

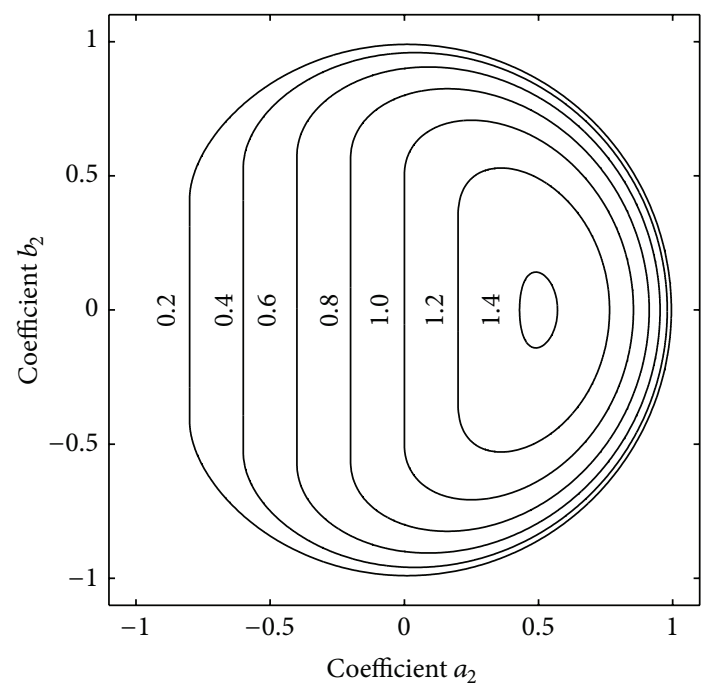

FIGURE 5: Contours of maximal absolute value of coefficients $a_{1}$ as a function of $a_{2}$ and $b_{2}$.

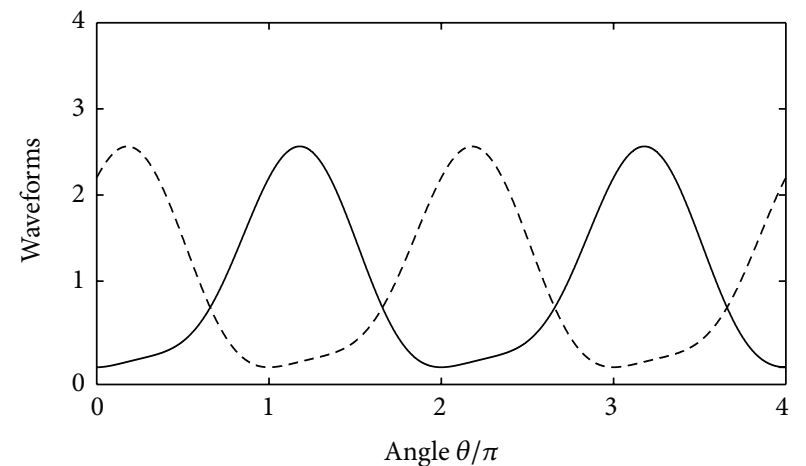

$$
\begin{aligned}
& a_{2}=0.1, b_{2}=0.3 \\
& -a_{1}=-1.1, b_{1}=-0.6, \theta_{0}=0 \\
& ---a_{1}=1.1, b_{1}=0.6, \theta_{0}=\pi
\end{aligned}
$$

FigURE 6: Nonnegative waveforms with maximal absolute value of coefficient $a_{1}, a_{2}=0.1$, and $b_{2}=0.3$.

Proof of Proposition 9. Let us consider nonnegative waveform $T_{2}(\theta)$ of type (1) with coefficients $a_{1}, b_{1}, a_{2}$, and $b_{2}$ expressed by (6)-(9) in terms of parameters $\xi, \psi, \varphi_{1}$, and $\varphi_{2}$.

According to (8) and (9), $a_{2}=b_{2}=0$ (i.e., $\left|A_{2}\right|=0$ ) implies $\cos \xi=0$ and/or $\sin 2 \psi=0$. For $\cos \xi=0$ from (6) it follows that $a_{1}=0$. On the other hand, $\sin 2 \psi=0$ implies either $\sin \psi=0$ or $\cos \psi=0$. For $\sin \psi=0$, according to (6)-(7), it follows that $a_{1}= \pm \sin 2 \xi \cos \varphi_{1}$ and $b_{1}= \pm \sin 2 \xi \sin \varphi_{1}$. For $\cos \psi=0$, according (6)-(7), it follows that $a_{1}= \pm \sin 2 \xi \cos \varphi_{2}$ and $b_{1}=\mp \sin 2 \xi \sin \varphi_{2}$. In both cases ( $\sin \psi=0$ and $\cos \psi=0$ ), maximal absolute value of $a_{1}$ is equal to 1 and corresponding coefficient $b_{1}$ is equal to zero. Consequently, if $a_{2}=b_{2}=0$, nonnegative waveform of type (1) with maximal absolute value of coefficient $a_{1}$ is either $T_{2}(\theta)=1-\cos \theta$ if $a_{1}=-1$ or $T_{2}(\theta)=1+\cos \theta$ if $a_{1}=1$. These 


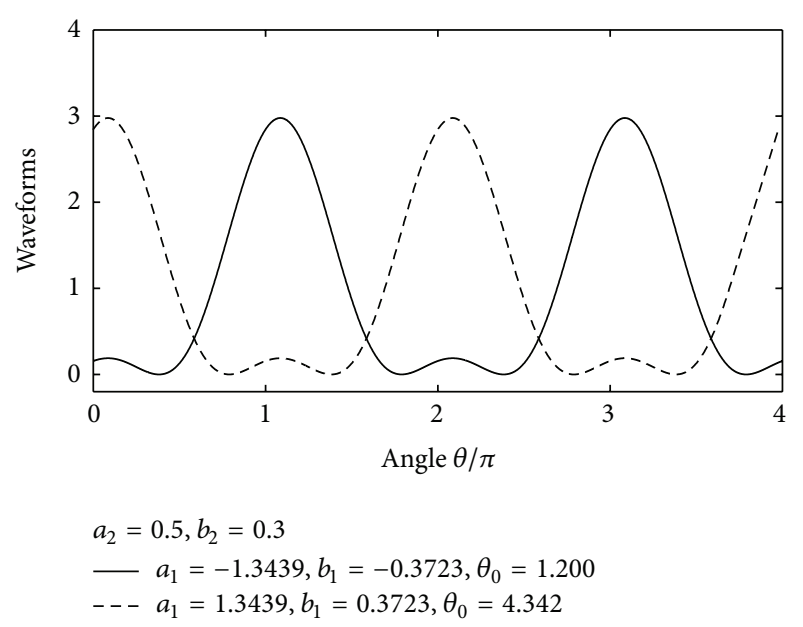

FigURE 7: Nonnegative waveforms with maximal absolute value of coefficient $a_{1}, a_{2}=0.5$, and $b_{2}=0.3$.

two waveforms coincide with (59) and (60) for $a_{2}=b_{2}=0$, respectively.

Suppose now that $\left|A_{2}\right| \neq 0$. According to (8)-(9) and (32), $a_{2}=A_{2} \cos \left(\varphi_{1}-\varphi_{2}\right)$ and $b_{2}=A_{2} \sin \left(\varphi_{1}-\varphi_{2}\right)$. Since $A_{2}$ does not depend on $\varphi_{1}$ and $\varphi_{2}$, it follows that $a_{2}$ and $b_{2}$ depend on $\varphi_{1}-\varphi_{2}$ only. Therefore, for prescribed $a_{2}$ and $b_{2}$, $\partial\left(\varphi_{1}-\varphi_{2}\right) / \partial \varphi_{1}=0$ which further implies that $\partial \varphi_{2} / \partial \varphi_{1}=$ 1. Consequently, in the course of finding maximal absolute value of coefficient $a_{1}$, we can set first derivative of $a_{1}$ in respect to $\varphi_{1}$ to zero. By using $\partial \varphi_{2} / \partial \varphi_{1}=1$ and $\partial a_{1} / \partial \varphi_{1}=0$, from (6), we obtain

$$
0=\sin 2 \xi\left(\cos \psi \sin \varphi_{1}+\sin \psi \sin \varphi_{2}\right) .
$$

Second derivative of $a_{1}$ with respect to $\varphi_{1}$ is equal to $-a_{1}$. It is positive (negative) if $a_{1}<0\left(a_{1}>0\right)$. Therefore, (69) lead to maximal absolute value of coefficient $a_{1}$. Substitution of $\sin 2 \xi \sin \psi \sin \varphi_{2}=-\sin 2 \xi \cos \psi \sin \varphi_{1}$ into (7) and (9) multiplied with $4 \sin ^{2} \xi$ yields

$$
b_{1}=2 \sin 2 \xi \cos \psi \sin \varphi_{1},
$$

$4 b_{2} \sin ^{2} \xi=2 \sin ^{2} 2 \xi \cos \psi \sin \varphi_{1}\left(\cos \psi \cos \varphi_{1}+\sin \psi \cos \varphi_{2}\right)$,

respectively. From (6), (70), and (71), it follows that

$$
a_{1} b_{1}=4 b_{2} \sin ^{2} \xi
$$

The sum of squared (6) and squared (69) equals

$$
a_{1}^{2}=\sin ^{2} 2 \xi\left(1+\sin 2 \psi \cos \left(\varphi_{1}-\varphi_{2}\right)\right) .
$$

From (8), it follows that (73) can be expressed as $a_{1}^{2}=$ $4 \sin ^{2} \xi\left(\cos ^{2} \xi+a_{2}\right)$. It can be rewritten in the following form:

$$
a_{1}^{2}=\left(1+a_{2}\right)^{2}-\left[2 \cos ^{2} \xi-\left(1-a_{2}\right)\right]^{2},
$$

where, according to (32), $\cos ^{2} \xi \geq\left|A_{2}\right|$. It is easy to see that, for prescribed $a_{2}, a_{1}^{2}$ is maximal when $\left[2 \cos ^{2} \xi-\left(1-a_{2}\right)\right]^{2}$ is minimal. Accordingly, in what follows, we will consider the following two cases: Case (i) $\cos ^{2} \xi=\left(1-a_{2}\right) / 2$ and Case (ii) $\cos ^{2} \xi=\left|A_{2}\right|>\left(1-a_{2}\right) / 2$.

Insertion of $\cos ^{2} \xi=\left(1-a_{2}\right) / 2$ (Case (i)) into (32) lead to $\sin 2 \psi=2 A_{2} /\left(1-a_{2}\right)$. Furthermore, $|\sin 2 \psi| \leq 1$ implies

$$
2\left|A_{2}\right| \leq 1-a_{2} \text {. }
$$

From $\cos ^{2} \xi=\left(1-a_{2}\right) / 2,(74),(70)$, and (72), we obtain

$$
\begin{gathered}
a_{1}^{2}=\left(1+a_{2}\right)^{2}, \\
b_{1}=\operatorname{sgn}\left(a_{1}\right) 2 b_{2},
\end{gathered}
$$

where sgn denotes sign function. Substitution of $a_{1}=-(1+$ $a_{2}$ ) and $b_{1}=-2 b_{2}$ into (1) lead to (59). Furthermore, substitution of $a_{1}=1+a_{2}$ and $b_{1}=2 b_{2}$ into (1) lead to (60).

In Case (ii) from $\cos ^{2} \xi=\left|A_{2}\right|$ and (74) it follows that $a_{1}^{2}=4\left(1-\left|A_{2}\right|\right)\left(\left|A_{2}\right|+a_{2}\right)$; that is,

$$
a_{1}=\operatorname{sgn}\left(a_{1}\right) 2 \sqrt{1-\left|A_{2}\right|} \sqrt{\left|A_{2}\right|+a_{2}} .
$$

Clearly, $\cos ^{2} \xi=\left|A_{2}\right|>\left(1-a_{2}\right) / 2$, that is,

$$
2\left|A_{2}\right|>1-a_{2}
$$

implies $1-\left|A_{2}\right|<\left|A_{2}\right|+a_{2}$, which, together with $\left|A_{2}\right| \leq 1$, further implies $\left|A_{2}\right|+a_{2} \neq 0$. Therefore, $a_{1}=0$ if and only if $\left|A_{2}\right|=1$. Since $a_{1}=0$ if and only if $\left|A_{2}\right|=1$ and $\sin ^{2} \xi=$ $1-\left|A_{2}\right|$, from (70), (72), and (77), it follows that

$$
b_{1}=\operatorname{sgn}\left(a_{1}\right) 2 b_{2} \frac{\sqrt{1-\left|A_{2}\right|}}{\sqrt{\left|A_{2}\right|+a_{2}}} .
$$

Notice that $b_{2}$ can be written as $b_{2}=\operatorname{sgn}\left(b_{2}\right)\left|b_{2}\right|=$ $\operatorname{sgn}\left(b_{2}\right) \sqrt{A_{2}^{2}-a_{2}^{2}}$, which, substituted in (79), yields

$$
b_{1}=\operatorname{sgn}\left(a_{1} b_{2}\right) 2 \sqrt{1-\left|A_{2}\right|} \sqrt{\left|A_{2}\right|-a_{2}} .
$$

Insertion of (77) and (80) into (1) lead to

$$
\begin{aligned}
T_{2}(\theta)= & 1+a_{2} \cos 2 \theta+b_{2} \sin 2 \theta \\
& +2 \operatorname{sgn}\left(a_{1}\right) \sqrt{1-\left|A_{2}\right|} \\
& \times\left(\sqrt{\left|A_{2}\right|+a_{2}} \cos \theta+\operatorname{sgn}\left(b_{2}\right) \sqrt{\left|A_{2}\right|-a_{2}} \sin \theta\right) .
\end{aligned}
$$

Since $a_{1}=0$ if and only if $\left|A_{2}\right|=1$, it follows that $1-\left|A_{2}\right|=$ $\left(\operatorname{sgn}\left(a_{1}\right) \sqrt{1-\left|A_{2}\right|}\right)^{2}$, which further implies that (81) can be expressed as

$$
\begin{gathered}
T_{2}(\theta)=2\left|A_{2}\right|\left(\operatorname{sgn}\left(a_{1}\right) \sqrt{\frac{1-\left|A_{2}\right|}{2\left|A_{2}\right|}}+\sqrt{\frac{\left|A_{2}\right|+a_{2}}{2\left|A_{2}\right|}} \cos \theta\right. \\
\left.+\operatorname{sgn}\left(b_{2}\right) \sqrt{\frac{\left|A_{2}\right|-a_{2}}{2\left|A_{2}\right|}} \sin \theta\right)^{2} .
\end{gathered}
$$


From $\left|A_{2}\right| \pm a_{2} \leq 2\left|A_{2}\right|$ and $\left|A_{2}\right|+a_{2}+\left|A_{2}\right|-a_{2}=2\left|A_{2}\right|$, it follows that (82) can be rewritten as

$$
T_{2}(\theta)=2\left|A_{2}\right|\left(\operatorname{sgn}\left(a_{1}\right) \sqrt{\frac{1-\left|A_{2}\right|}{2\left|A_{2}\right|}}+\cos (\theta-\alpha)\right)^{2},
$$

where

$$
\begin{gathered}
\cos \alpha=\sqrt{\frac{\left|A_{2}\right|+a_{2}}{2\left|A_{2}\right|}}, \\
\sin \alpha=\operatorname{sgn}\left(b_{2}\right) \sqrt{\frac{\left|A_{2}\right|-a_{2}}{2\left|A_{2}\right|}} .
\end{gathered}
$$

Relation (78) implies $2\left|A_{2}\right|>1-\left|A_{2}\right|$ and therefore $T_{2}\left(\theta_{0}\right)=$ 0 for some $\theta_{0}$. From (83),

$$
\cos \left(\theta_{0}-\alpha\right)=-\operatorname{sgn}\left(a_{1}\right) \sqrt{\frac{1-\left|A_{2}\right|}{2\left|A_{2}\right|}} .
$$

Substitution of (85) into (83) yields $T_{2}(\theta)=$ $2\left|A_{2}\right|\left[\cos (\theta-\alpha)-\cos \left(\theta_{0}-\alpha\right)\right]^{2}$, which can be rewritten as

$$
T_{2}(\theta)=2\left|A_{2}\right|\left[1-\cos \left(\theta-\theta_{0}\right)\right]\left[1-\cos \left(\theta+\theta_{0}-2 \alpha\right)\right] .
$$

Replacing $\alpha$ by $\left(\theta_{0}+\theta_{0}^{\prime}\right) / 2$ in (84)-(86), where $\theta_{0}^{\prime}$ is new variable, immediately lead to (61) and (62).

Finally, for $2\left|A_{2}\right|=1-a_{2}$ and $a_{1} \leq 0$, from (62), it follows that $\cos \theta_{0}=1$ or $\cos \theta_{0}^{\prime}=1$. For $\cos \theta_{0}=1$, according to (62), it follows that $\cos \theta_{0}^{\prime}=a_{2} /\left|A_{2}\right|$ and $\sin \theta_{0}^{\prime}=b_{2} /\left|A_{2}\right|$. Consequently, (61) coincides with (59) for $2\left|A_{2}\right|=1-a_{2}$ and $a_{1} \leq 0$. Similarly, for $2\left|A_{2}\right|=1-a_{2}$ and $a_{1} \geq 0$, from (62), it follows that $\cos \theta_{0}=-1, \cos \theta_{0}^{\prime}=-a_{2} /\left|A_{2}\right|$, and $\sin \theta_{0}^{\prime}=$ $-b_{2} /\left|A_{2}\right|$. Consequently, (61) coincides with (60) for $2\left|A_{2}\right|=$ $1-a_{2}$ and $a_{1} \geq 0$. This completes the proof.

\section{Nonnegative Waveforms with at Least One Zero}

In this section, we provide general description of nonnegative waveforms of type (1) with at least one zero, that is, waveforms $T_{2}(\theta)$ for which $T_{2}(\theta) \geq 0$ and $T_{2}\left(\theta_{0}\right)=0$ for some $\theta_{0}$. Notice that conditions $T_{2}(\theta) \geq 0$ and $T_{2}\left(\theta_{0}\right)=0$ imply that $T_{2}^{\prime}\left(\theta_{0}\right)=0$. This type of nonnegative waveforms have proved to be of particular interest in PA design (e.g., see [1,3-7]). We also provide an algorithm for calculation of coefficients $a_{1}$ and $b_{1}$ of nonnegative waveforms with at least one zero and prescribed coefficients $a_{2}$ and $b_{2}$ (Section 5.1).

Proposition 12. Every nonnegative waveform of type (1) with at least one zero can be expressed in the following form:

$$
T_{2}(\theta)=\frac{2\left[1-\cos \left(\theta-\theta_{0}\right)\right]\left[1-\sin 2 \psi \cos \left(\theta+\theta_{0}+\beta\right)\right]}{2+\sin 2 \psi \cos \left(2 \theta_{0}+\beta\right)},
$$

where $\theta_{0}, \psi$, and $\beta$ are arbitrary real numbers.
Proof. Notice that (12) can be rewritten as

$$
\begin{aligned}
T_{2}(\theta)= & {\left[\operatorname{Re}\left\{\underline{H}_{2}\left(e^{j \theta}\right)\right\} \cos \theta+\operatorname{Im}\left\{\underline{H}_{2}\left(e^{j \theta}\right)\right\} \sin \theta\right]^{2} } \\
& +\left[\operatorname{Re}\left\{\underline{H}_{2}\left(e^{j \theta}\right)\right\} \sin \theta-\operatorname{Im}\left\{\underline{H}_{2}\left(e^{j \theta}\right)\right\} \cos \theta\right]^{2},
\end{aligned}
$$

which, after inserting (10), yields

$$
\begin{aligned}
T_{2}(\theta)= & {\left[\left(c_{0}+c_{2}\right) \cos \theta+\left(d_{0}-d_{2}\right) \sin \theta+c_{1}\right]^{2} } \\
& +\left[\left(c_{0}-c_{2}\right) \sin \theta-\left(d_{0}+d_{2}\right) \cos \theta-d_{1}\right]^{2} .
\end{aligned}
$$

Suppose that $T_{2}\left(\theta_{0}\right)=0$ for some $\theta=\theta_{0}$. Then, for $\theta=\theta_{0}$, both squared terms in (89) are equal to zero; that is,

$$
\begin{aligned}
& c_{1}=-\left(c_{0}+c_{2}\right) \cos \theta_{0}-\left(d_{0}-d_{2}\right) \sin \theta_{0}, \\
& d_{1}=-\left(d_{0}+d_{2}\right) \cos \theta_{0}+\left(c_{0}-c_{2}\right) \sin \theta_{0} .
\end{aligned}
$$

Substituting (90) into (89) and taking into account that $\cos ^{2} \xi$, $a_{2}$, and $b_{2}$ are given by (18), (14), and (16), respectively, we obtain

$$
\begin{aligned}
T_{2}(\theta)= & \cos ^{2} \xi\left[\left(\cos \theta-\cos \theta_{0}\right)^{2}+\left(\sin \theta-\sin \theta_{0}\right)^{2}\right] \\
& +a_{2}\left[\left(\cos \theta-\cos \theta_{0}\right)^{2}-\left(\sin \theta-\sin \theta_{0}\right)^{2}\right] \\
& +b_{2}\left[\left(\cos \theta-\cos \theta_{0}\right)\left(\sin \theta-\sin \theta_{0}\right)\right] .
\end{aligned}
$$

It is easy to show that all terms in (91) have common factor $2\left[1-\cos \left(\theta-\theta_{0}\right)\right]$ and therefore $T_{2}(\theta)$ can be written as

$$
\begin{aligned}
T_{2}(\theta)= & 2\left[1-\cos \left(\theta-\theta_{0}\right)\right] \\
& \times\left[\cos ^{2} \xi-a_{2} \cos \left(\theta+\theta_{0}\right)-b_{2} \sin \left(\theta+\theta_{0}\right)\right] .
\end{aligned}
$$

Substituting (21)-(24) into (90) and then inserting resulting relations into (17), we obtain

$$
\cos ^{2} \xi=\left[2+\sin 2 \psi \cos \left(2 \theta_{0}+\phi_{2}-\phi_{1}\right)\right]^{-1} .
$$

Let us denote

$$
\beta=\phi_{2}-\phi_{1}
$$

Inserting (27)-(28) into (92), by using (93)-(94), we finally obtain (87).

Remark 13. Conversion of (87) into an additive form and comparison with (1) immediately lead to the explicit form of coefficients of nonnegative waveforms with at least one zero:

$$
\begin{aligned}
& a_{1}=-\frac{2\left(\sin 2 \psi \cos \left(\theta_{0}+\beta\right)+\cos \theta_{0}\right)}{2+\sin 2 \psi \cos \left(2 \theta_{0}+\beta\right)}, \\
& b_{1}=\frac{2\left(\sin 2 \psi \sin \left(\theta_{0}+\beta\right)-\sin \theta_{0}\right)}{2+\sin 2 \psi \cos \left(2 \theta_{0}+\beta\right)}, \\
& a_{2}=\frac{\sin 2 \psi \cos \beta}{2+\sin 2 \psi \cos \left(2 \theta_{0}+\beta\right)}, \\
& b_{2}=-\frac{\sin 2 \psi \sin \beta}{2+\sin 2 \psi \cos \left(2 \theta_{0}+\beta\right)} .
\end{aligned}
$$


According to (95) and (96), amplitude of fundamental harmonic is

$$
\left|A_{1}\right|=\frac{2 \sqrt{1+2 \sin 2 \psi \cos \left(2 \theta_{0}+\beta\right)+\sin ^{2} 2 \psi}}{2+\sin 2 \psi \cos \left(2 \theta_{0}+\beta\right)} .
$$

Also, according to (97) and (98), amplitude of second harmonic is

$$
\left|A_{2}\right|=\frac{|\sin 2 \psi|}{2+\sin 2 \psi \cos \left(2 \theta_{0}+\beta\right)} .
$$

Remark 14. Coefficients (95)-(98) of nonnegative waveforms with at least one zero can be obtained from coefficients (6)(9) of general case of nonnegative waveforms by substituting $\beta=\phi_{2}-\phi_{1}=\varphi_{2}-\varphi_{1}$ (see (94) and (30)), $\cos ^{2} \xi=$ $\left[2+\sin 2 \psi \cos \left(2 \theta_{0}+\beta\right)\right]^{-1}$ (see (93)-(94)), and

$$
\begin{aligned}
\sin 2 \xi \cos \varphi_{1} & =\frac{-2\left[\cos \psi \cos \theta_{0}+\sin \psi \cos \left(\theta_{0}+\beta\right)\right]}{2+\sin 2 \psi \cos \left(2 \theta_{0}+\beta\right)}, \\
\sin 2 \xi \sin \varphi_{1} & =\frac{-2\left[\cos \psi \sin \theta_{0}-\sin \psi \sin \left(\theta_{0}+\beta\right)\right]}{2+\sin 2 \psi \cos \left(2 \theta_{0}+\beta\right)} \\
\sin 2 \xi \cos \varphi_{2} & =\frac{-2\left[\sin \psi \cos \theta_{0}+\cos \psi \cos \left(\theta_{0}+\beta\right)\right]}{2+\sin 2 \psi \cos \left(2 \theta_{0}+\beta\right)} \\
\sin 2 \xi \sin \varphi_{2} & =\frac{2\left[\sin \psi \sin \theta_{0}-\cos \psi \sin \left(\theta_{0}+\beta\right)\right]}{2+\sin 2 \psi \cos \left(2 \theta_{0}+\beta\right)}
\end{aligned}
$$

Remark 15. In Section 3 (see Proposition 4 and Remark 5), we show that every nonnegative waveform of type (1) with maximal amplitude of fundamental harmonic for prescribed second harmonic amplitude has at least one zero. Coefficients (42) of waveforms with maximal amplitude of fundamental harmonic for prescribed $\left|A_{2}\right|$, when $0 \leq\left|A_{2}\right| \leq 1 / 3$, can be obtained from (95)-(98) by replacing $\sin 2 \psi$ with $|\sin 2 \psi|$ and $\beta$ with $-2 \theta_{0}$. Similarly, coefficients (43) of waveforms with maximal amplitude of fundamental harmonic for prescribed $\left|A_{2}\right|$, when $1 / 3 \leq\left|A_{2}\right| \leq 1$, can be obtained from (95)-(98) by replacing $\sin 2 \psi$ with 1 and $\beta$ with $-\left(\theta_{0}+\theta_{0}^{\prime}\right)$.

From the above consideration, it is easy to see that among nonnegative waveforms with at least one zero and prescribed second harmonic amplitude, there are waveforms for which the amplitude of fundamental harmonic is not maximal (e.g., see Figures 9 and 10).

Remark 16. In Section 4 (see Proposition 9 and Remark 10), we show that every nonnegative waveform of type (1) with maximal absolute value of coefficient $a_{1}$ for prescribed coefficients $a_{2}$ and $b_{2}$ has at least one zero. Coefficients (63) of waveforms with maximal coefficient $a_{1}$, when $2\left|A_{2}\right| \leq$ $1-a_{2}$, can be obtained from (95)-(98) by setting $\theta_{0}=$ $\pi$. Similarly, coefficients (64) of waveforms with minimal coefficient $a_{1}$ (also maximal absolute value), when $2\left|A_{2}\right| \leq$ $1-a_{2}$, can be obtained from (95)-(98) by setting $\theta_{0}=0$. Also, coefficients (67)-(68) of waveforms with maximal absolute value of coefficient $a_{1}$, when $2\left|A_{2}\right|>1-a_{2}$, can be obtained from (95)-(98) by replacing $\sin 2 \psi$ with 1 and $\beta$ with $-\left(\theta_{0}+\right.$ $\left.\theta_{0}^{\prime}\right)$.

From the above consideration, it is easy to see that among nonnegative waveforms with at least one zero and prescribed coefficients $a_{2}$ and $b_{2}$, there are waveforms for which absolute value of coefficient $a_{1}$ is not maximal (e.g., see Figures 9 and 10).

5.1. Nonnegative Waveforms with Prescribed Second Harmonic Coefficients. In this subsection, an algorithm for calculation of fundamental harmonic coefficients of nonnegative waveforms with at least one zero for prescribed second harmonic coefficients is provided. The range of instance $\theta_{0}$ at which waveform takes zero value depends on the chosen pair $a_{2}$ and $b_{2}$.

Let us show first that $\left|A_{2}\right|=1$ lead to $a_{1}=b_{1}=0$. According to (100), $\left|A_{2}\right|=1$ implies $|\sin 2 \psi|=1$ and $\sin 2 \psi \cos \left(2 \theta_{0}+\beta\right)=-1$. These relations from (99) further imply $\left|A_{1}\right|=0$; that is, $a_{1}=b_{1}=0$. Therefore, in what follows we will consider only those pairs $\left(a_{2}, b_{2}\right)$ for which $\left|A_{2}\right|<1$.

Solving (97)-(98) in terms of $\sin 2 \psi \cos \beta$ and $\sin 2 \psi \sin \beta$, we obtain

$$
\begin{aligned}
& \sin 2 \psi \cos \beta=\frac{2 a_{2}\left(1+\tan ^{2} \theta_{0}\right)}{\left(1+a_{2}\right) \tan ^{2} \theta_{0}-2 b_{2} \tan \theta_{0}+1-a_{2}}, \\
& \sin 2 \psi \sin \beta=\frac{-2 b_{2}\left(1+\tan ^{2} \theta_{0}\right)}{\left(1+a_{2}\right) \tan ^{2} \theta_{0}-2 b_{2} \tan \theta_{0}+1-a_{2}},
\end{aligned}
$$

where range of $\theta_{0}$ depends on the chosen pair $\left(a_{2}, b_{2}\right)$.

A good part of this Subsection is devoted to finding this range. In this context, let us begin with substitution of (102) into $(\sin 2 \psi \cos \beta)^{2}+(\sin 2 \psi \sin \beta)^{2} \leq 1$. This lead to $\left(4 a_{2}^{2}+\right.$ $\left.4 b_{2}^{2}\right)\left(1+\tan ^{2} \theta_{0}\right)^{2} \leq\left[\left(1+a_{2}\right) \tan ^{2} \theta_{0}-2 b_{2} \tan \theta_{0}+1-a_{2}\right]^{2}$ or equivalently to

$$
\begin{aligned}
& {\left[\left(2\left|A_{2}\right|+1+a_{2}\right) \tan ^{2} \theta_{0}-2 b_{2} \tan \theta_{0}+2\left|A_{2}\right|+1-a_{2}\right]} \\
& \cdot\left[\left(2\left|A_{2}\right|-1-a_{2}\right) \tan ^{2} \theta_{0}+2 b_{2} \tan \theta_{0}+2\left|A_{2}\right|-1\right. \\
& \left.\quad+a_{2}\right] \leq 0 .
\end{aligned}
$$

The expressions in the square brackets can be considered as second degree polynomials in terms of $\tan \theta_{0}$ with the following discriminants:

$$
\begin{gathered}
D_{1}=-4\left(3 A_{2}^{2}+4\left|A_{2}\right|+1\right), \\
D_{2}=4\left(3\left|A_{2}\right|-1\right)\left(1-\left|A_{2}\right|\right),
\end{gathered}
$$

respectively. Since $D_{1}<0$ and $2\left|A_{2}\right|+1+a_{2}>0$ for all $\left(a_{2}, b_{2}\right)$, it follows that first factor is always positive. Therefore, inequality (103) is equivalent to

$$
\left(1+a_{2}-2\left|A_{2}\right|\right) \tan ^{2} \theta_{0}-2 b_{2} \tan \theta_{0}+1-a_{2}-2\left|A_{2}\right| \geq 0 .
$$


Consequently, the problem of finding range of $\tan \theta_{0}$, for which (102) holds, can be reformulated as the problem of finding those $\tan \theta_{0}$ for which inequality (106) holds. The discussion involves discriminant $D_{2}$ (see (105)) and leading term coefficient

$$
\lambda=1+a_{2}-2\left|A_{2}\right| .
$$

In what follows, we first consider all cases with $\lambda \neq 0$. Let $x_{1}$ and $x_{2}$ be the roots of second degree polynomial on the left side of (106); that is,

$$
\begin{aligned}
& x_{1}=\frac{b_{2}-\sqrt{\left(3\left|A_{2}\right|-1\right)\left(1-\left|A_{2}\right|\right)}}{1+a_{2}-2\left|A_{2}\right|}, \\
& x_{2}=\frac{b_{2}+\sqrt{\left(3\left|A_{2}\right|-1\right)\left(1-\left|A_{2}\right|\right)}}{1+a_{2}-2\left|A_{2}\right|} .
\end{aligned}
$$

According to (105) and (107), $\left|A_{2}\right|<1 / 3$ implies $D_{2}<0$ and $\lambda>0$ (see Figure 8). Also $\left|A_{2}\right|=1 / 3$ and $2\left|A_{2}\right| \neq 1+a_{2}$ imply $D_{2}=0$ and $2\left|A_{2}\right|<1+a_{2}(\lambda>0)$. Similarly, $\left|A_{2}\right|>1 / 3$ and $2\left|A_{2}\right|<1+a_{2}$ imply $D_{2}>0$ and $\lambda>0$. Notice that $2\left|A_{2}\right|>1+a_{2}(\lambda<0)$ implies $\left|A_{2}\right|>1 / 3$, which further implies $D_{2}>0$, Thus, for $\lambda \neq 0$ :

(1) if $\left|A_{2}\right|<1 / 3$, then (106) holds for every $\tan \theta_{0}$,

(2) if $\left|A_{2}\right|=1 / 3$ and $2\left|A_{2}\right| \neq 1+a_{2}$, then (106) holds for every $\tan \theta_{0}$,

(3) if $\left|A_{2}\right|>1 / 3$ and $2\left|A_{2}\right|<1+a_{2}$, then (106) holds for

$$
\tan \theta_{0} \leq x_{1} \quad \text { or } \tan \theta_{0} \geq x_{2},
$$

(4) if $2\left|A_{2}\right|>1+a_{2}$, then (106) holds for

$$
x_{2} \leq \tan \theta_{0} \leq x_{1},
$$

where $x_{1}$ and $x_{2}$ are given by (108).

For $2\left|A_{2}\right|=1+a_{2}$, (106) reduces to $b_{2} \tan \theta_{0} \leq-a_{2}$. Thus, for $\lambda=0$, we have another two cases:

(5) if $2\left|A_{2}\right|=1+a_{2}$ and $b_{2} \neq 0$, then (106) holds for

$$
\begin{aligned}
& \tan \theta_{0} \leq-\frac{a_{2}}{b_{2}} \quad \text { if } b_{2}>0, \\
& \tan \theta_{0} \geq-\frac{a_{2}}{b_{2}} \quad \text { if } b_{2}<0,
\end{aligned}
$$

(6) if $2\left|A_{2}\right|=1+a_{2}$ and $b_{2}=0$, then (106) holds for every $\tan \theta_{0}$.

Notice that $2\left|A_{2}\right|=1+a_{2}$ and $b_{2} \neq 0$ imply $\left|A_{2}\right|>1 / 3$, whereas $2\left|A_{2}\right|=1+a_{2}$ and $b_{2}=0$ imply $\left|A_{2}\right|=1 / 3$ (see also Figure 8). Therefore, Cases (1), (2), and (6) can be merged into one case which reads the following: if $\left|A_{2}\right| \leq 1 / 3$, then (106) holds for every $\tan \theta_{0}$. In all other cases, namely, Cases

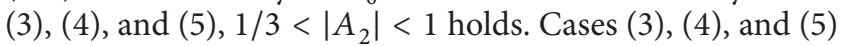
correspond to $2\left|A_{2}\right|<1+a_{2}, 2\left|A_{2}\right|>1+a_{2}$, and $2\left|A_{2}\right|=1+a_{2}$, respectively.
To obtain closed form expressions for coefficients $a_{1}$ and $b_{1}$ of nonnegative waveforms with zero at $\theta_{0}$ and prescribed coefficients $a_{2}$ and $b_{2}$ it is sufficient to substitute (102) into (95)-(96):

$$
\begin{aligned}
& a_{1}=-\cos ^{3} \theta_{0}\left[2 b_{2} \tan ^{3} \theta_{0}+\left(3 a_{2}+1\right) \tan ^{2} \theta_{0}+\left(1+a_{2}\right)\right], \\
& b_{1}=\cos ^{3} \theta_{0}\left[-2 b_{2}+\left(3 a_{2}-1\right) \tan \theta_{0}-\left(1-a_{2}\right) \tan ^{3} \theta_{0}\right],
\end{aligned}
$$

where range of $\tan \theta_{0}$ is discussed earlier in this subsection (see Cases (1)-(6)). For $\cos \theta_{0}=0$, expressions (112)-(113) reduce to $a_{1}=-2 b_{2} \sin ^{3} \theta_{0}$ and $b_{1}=-\left(1-a_{2}\right) \sin ^{3} \theta_{0}$.

Since the range of $\theta_{0}$ depends on the choice of pair $\left(a_{2}, b_{2}\right)$ we provide an algorithm to facilitate calculation of coefficients $a_{1}$ and $b_{1}$ of nonnegative waveforms with at least one zero for prescribed coefficients $a_{2}$ and $b_{2}$, providing that $\left|A_{2}\right|<1$.

Algorithm 17. One has the following steps:

(i) calculate $\left|A_{2}\right|=\sqrt{a_{2}^{2}+b_{2}^{2}}$;

(ii) if $\left|A_{2}\right| \leq 1 / 3$, then $\tan \theta_{0}$ can take any value; else, if $2\left|A_{2}\right|<1+a_{2}$, calculate $x_{1}$ and $x_{2}$ from (108) and calculate range of $\tan \theta_{0}$ according to (109);

else, if $2\left|A_{2}\right|>1+a_{2}$ calculate $x_{1}$ and $x_{2}$ from (108) and calculate range of $\tan \theta_{0}$ according to (110);

else, calculate range of $\tan \theta_{0}$ according to (111);

(iii) choose $\theta_{0}$ such that $\tan \theta_{0}$ belongs to the range calculated in previous step;

(iv) if $\sin \theta_{0}= \pm 1$, then calculate $a_{1}=-2 b_{2} \sin \theta_{0}$ and $b_{1}=-\left(1-a_{2}\right) \sin \theta_{0}$;

else, calculate $a_{1}$ and $b_{1}$ according to (112) and (113), respectively.

As an example, nonnegative waveforms with at least one zero, $a_{2}=0.1$, and $b_{2}=0.2$ are presented in Figure 9 for the following three choices of $\theta_{0}: \theta_{0}=\pi, \theta_{0}=4 \pi / 3$, and $\theta_{0}=7 \pi / 4$. Since in this case $\left|A_{2}\right|<1 / 3$, according to step (ii) of Algorithm 17, $\theta_{0}$ can be chosen without any restrictions. Another three examples of nonnegative waveforms with at least one zero, $a_{2}=0.1$ and $b_{2}=0.4$ are presented in Figure 10 for the following choices of $\theta_{0}: \theta_{0}=4.3702, \theta_{0}=2.7994$, and $\theta_{0}=-1.4736$. Since in this case $\left|A_{2}\right|>1 / 3$ and $2\left|A_{2}\right|<1+a_{2}$, according to step (ii) of Algorithm 17, $\theta_{0}$ can be chosen so that either $\tan \theta_{0} \leq 0.0975$ or $\tan \theta_{0} \geq 2.8076$.

\section{Nonnegative Cosine Waveforms}

Nonnegative cosine waveforms play an important role in PA modelling (e.g., see [7]). In this section, we provide general description of nonnegative cosine waveforms as well as nonnegative cosine waveforms with at least one zero. We show that nonnegative cosine waveforms with at least one zero coincide with nonnegative cosine waveforms with maximal absolute value of coefficient $a_{1}$ for prescribed coefficient $a_{2}$. Few examples of shapes of cosine waveforms are presented in Figure 11. 


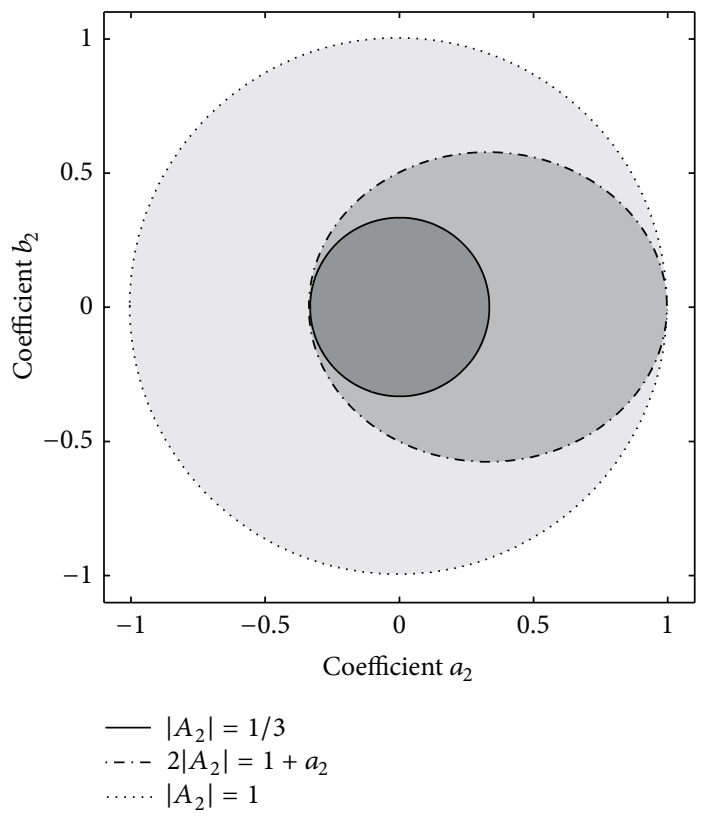

Figure 8: Circle $\left|A_{2}\right|=1 / 3$, ellipse $2\left|A_{2}\right|=1+a_{2}$, and circle $\left|A_{2}\right|=$ 1.

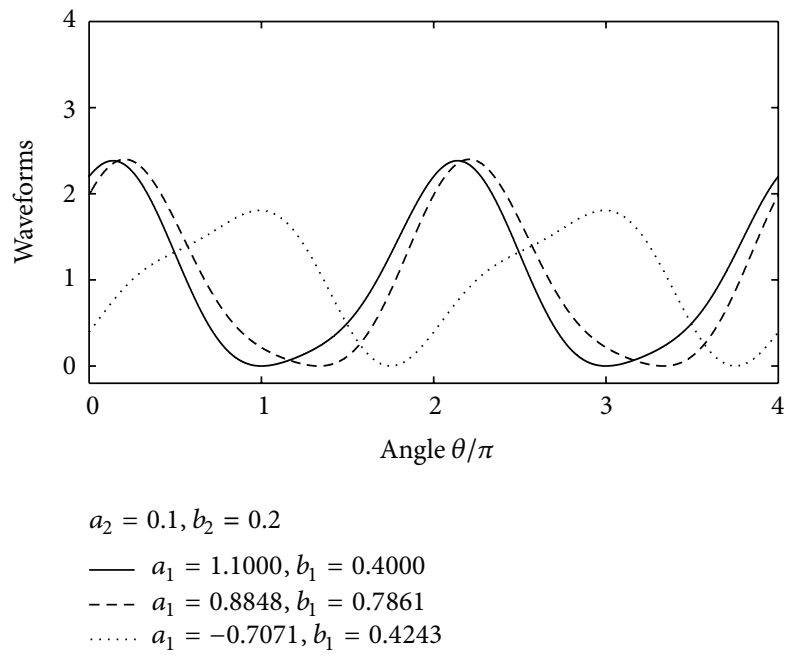

FIGURE 9: Nonnegative waveforms with one zero, $a_{2}=0.1$, and $b_{2}=$ 0.2 .

According to [8], every nonnegative cosine polynomial (nonnegative trigonometric polynomial of type (1) with $b_{1}=$ $b_{2}=0$ ) can be obtained from (10)-(12) by setting $d_{0}=d_{1}=$ $d_{2}=0$. Substitution of $d_{0}=d_{1}=d_{2}=0$ into (89) lead to the following description of nonnegative cosine polynomials:

$$
T_{2}(\theta)=\left[\left(c_{0}+c_{2}\right) \cos \theta+c_{1}\right]^{2}+\left[\left(c_{0}-c_{2}\right) \sin \theta\right]^{2},
$$

where $c_{0}, c_{1}$, and $c_{2}$ satisfy the following constraint:

$$
c_{0}^{2}+c_{1}^{2}+c_{2}^{2}=1
$$

According to (20), (23), and (24), conditions $d_{0}=d_{1}=d_{2}=0$ in terms of parameters $\xi, \vartheta, \psi, \phi_{1}$, and $\phi_{2}$ can be rewritten

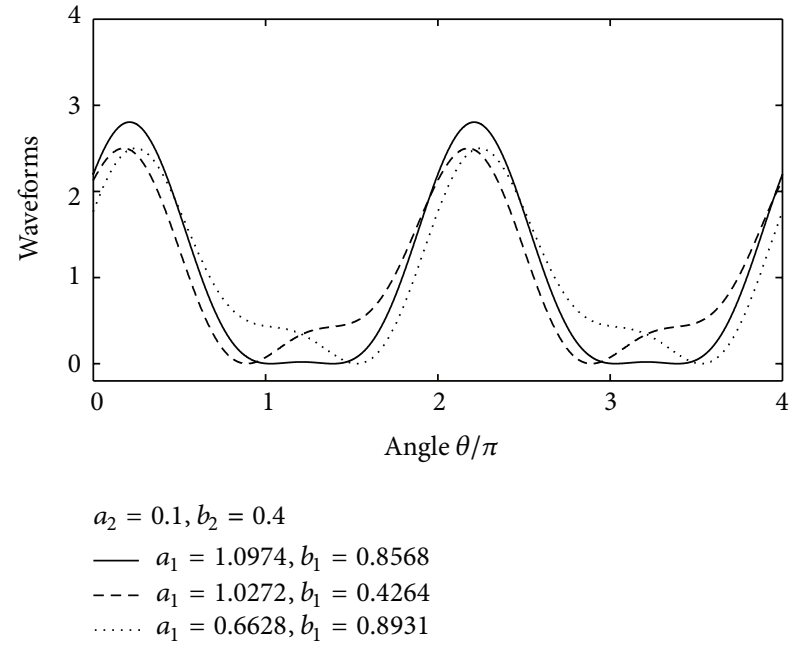

FIGURE 10: Nonnegative waveforms with at least one zero, $a_{2}=0.1$, and $b_{2}=0.4$.

as $\sin \vartheta=\sin \phi_{1}=\sin \phi_{2}=0$. Clearly, they are fulfilled, for example, for $\vartheta=\phi_{1}=\phi_{2}=0$. Thus, according to (19), (21), and (22), parameters $c_{1}, c_{0}$, and $c_{2}$ become $c_{1}=$ $\sin \xi, c_{0}=\cos \xi \cos \psi$, and $c_{2}=\cos \xi \sin \psi$. Inserting these expressions for $c_{0}, c_{1}$, and $c_{2}$ into (114) lead to the following form of nonnegative cosine polynomials:

$$
\begin{aligned}
T_{2}(\theta)= & {[\cos \xi(\cos \psi+\sin \psi) \cos \theta+\sin \xi]^{2} } \\
& +[\cos \xi(\cos \psi-\sin \psi) \sin \theta]^{2},
\end{aligned}
$$

which coefficients are

$$
\begin{aligned}
& a_{1}=\sin 2 \xi(\cos \psi+\sin \psi), \\
& a_{2}=\cos ^{2} \xi \sin 2 \psi .
\end{aligned}
$$

Expressions (117) provide general description of nonnegative cosine waveforms in terms of two independent parameters: $\xi$ and $\psi$.

Description of nonnegative cosine waveforms with at least one zero is presented in Proposition 18.

Proposition 18. Each nonnegative cosine waveform up to second harmonic with at least one zero can be represented in one of the following forms:

$$
\begin{aligned}
& T_{2}(\theta)=\frac{2[1-\cos \theta][1-\sin 2 \psi \cos \theta]}{2+\sin 2 \psi}, \\
& T_{2}(\theta)=\frac{2[1+\cos \theta][1+\sin 2 \psi \cos \theta]}{2+\sin 2 \psi}, \\
& T_{2}(\theta)=\frac{2\left[1-\cos \left(\theta-\theta_{0}\right)\right]\left[1-\cos \left(\theta+\theta_{0}\right)\right]}{2+\cos 2 \theta_{0}} .
\end{aligned}
$$

Remark 19. All nonnegative cosine waveforms up to second harmonic with at least one zero, except three of them, can 


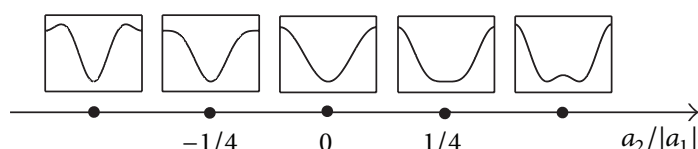

FIGURE 11: Shapes of cosine polynomials up to second harmonic.

be represented in exactly one of forms (118)-(120). For these three exceptions, there are two possible forms:

(i) waveform for which $\sin 2 \psi=-1$ can be expressed in both forms (118) and (119);

(ii) waveform for which $\sin 2 \psi=1$ and $\theta_{0}=0$ can be expressed in both forms (118) and (120);

(iii) waveform for which $\sin 2 \psi=1$ and $\theta_{0}=\pi$ can be expressed in both forms (119) and (120).

Proof of Proposition 18. We shall first consider nonnegative waveforms of type (1) with at least one zero for which $b_{2}=0$ and then include additional condition $b_{1}=0$. All such waveforms can be obtained from (87), (96), and (98). Thus, according to (98), $b_{2}=0$ implies $\sin 2 \psi \sin \beta=0$, which further lead to $\sin 2 \psi \cos \beta= \pm \sin 2 \psi$. Suppose that $\sin 2 \psi \cos \beta=\sin 2 \psi$. Substitution of $\sin 2 \psi \sin \beta=0$ and $\sin 2 \psi \cos \beta=\sin 2 \psi$ into (96) and setting $b_{1}=0$ imply $(1-\sin 2 \psi) \sin \theta_{0}=0$. Furthermore, substitution of $\theta_{0}=$ $0, \theta_{0}=\pi$ and $\sin 2 \psi=1$ into (87) immediately lead to (118), (119), and (120), respectively. The other case when $\sin 2 \psi \cos \beta=-\sin 2 \psi$ lead to the same class of nonnegative cosine waveforms with at least one zero, since substitution of $\psi$ by $-\psi$, maps the family of polynomials into itself. This completes the proof.

Coefficients $a_{1}$ and $a_{2}$ of nonnegative cosine waveforms with at least one zero can be expressed in terms of parameters $\psi$ or $\theta_{0}$ by converting (118)-(120) into additive form (1). In what follows, by eliminating $\psi$ or $\theta_{0}$, we provide relations between coefficients $a_{1}$ and $a_{2}$.

Conversion of (118) into an additive form lead to $a_{2}=$ $\sin 2 \psi /(2+\sin 2 \psi)$ and $a_{1}=-\left(1+a_{2}\right)$. First relation implies $-1 \leq a_{2} \leq 1 / 3$ and therefore, for coefficients of waveforms (118), we obtain

$$
a_{1}=-\left(1+a_{2}\right) \text { for }-1 \leq a_{2} \leq \frac{1}{3} .
$$

Conversion of (119) into an additive form lead to $a_{2}=$ $\sin 2 \psi /(2+\sin 2 \psi)$ and $a_{1}=1+a_{2}$. Therefore, for coefficients of waveforms (119), the following relations hold:

$$
a_{1}=1+a_{2} \text { for }-1 \leq a_{2} \leq \frac{1}{3} .
$$

Conversion of (120) into an additive form lead to $a_{2}=1 /(2+$ $\left.\cos 2 \theta_{0}\right)$ and $a_{1}=-4 a_{2} \cos \theta_{0}$. First relation implies $1 / 3 \leq$ $a_{2} \leq 1$ and $\cos \theta_{0}= \pm \sqrt{\left(1-a_{2}\right) /\left(2 a_{2}\right)}$. Substituting $\cos \theta_{0}$ into $a_{1}$ provides $a_{1}$ in terms of $a_{2}$. Consequently, for coefficients of waveforms (120), the following relations hold:

$$
a_{1}= \pm \sqrt{8 a_{2}\left(1-a_{2}\right)} \text { for } \frac{1}{3} \leq a_{2} \leq 1
$$

It is easy to see that expressions (121)-(123) can be obtained from (63), (64), (67), (68), by setting $b_{2}=0,\left|A_{2}\right|=$ $\left|a_{2}\right|$ and $\left|A_{2}\right|=a_{2}$ when $a_{2} \geq 0$. Therefore, every nonnegative cosine waveform with at least one zero has maximal absolute value of coefficient $a_{1}$ for prescribed coefficient $a_{2}$.

Every cosine polynomial corresponds to a pair of real numbers $\left(a_{1}, a_{2}\right)$ and vice versa. In Figure 12, grey area consists of those pairs $\left(a_{1}, a_{2}\right)$ that correspond to nonnegative cosine polynomials. The boundary of this area corresponds to nonnegative cosine waveforms with at least one zero, described by relations (121)-(123). A number of shapes of nonnegative cosine waveforms with at least one zero, plotted on interval $[-\pi, \pi]$ are also presented in Figure 12. Cosine polynomial that can be expressed in both forms (118) and (119) corresponds to the common point of line segments (121) and (122). This common point is the cusp in Figure 12, with coordinates $a_{1}=0$ and $a_{2}=-1$. Cosine polynomial that can be expressed in both forms (118) and (120) corresponds to the common point of line segments (121) and (123), whereas one that can be expressed in both forms (119) and (120) corresponds to the common point of segments (122) and (123). The last two points, represented with white circle dots in Figure 12, correspond to the so-called maximally flat waveforms with coefficients $a_{1}= \pm 4 / 3$ and $a_{2}=1 / 3$ (e.g., see [5]). White triangle dots $( \pm \sqrt{2}, 1 / 2)$ refer to the cosine polynomials with maximal amplitude of fundamental harmonic.

\section{Three Case Studies of the Usage of Description of Nonnegative Waveforms in PA Efficiency Analysis}

This section provides three case studies of the usage of descriptions of nonnegative waveforms introduced above, for the purpose of waveforms modelling in the PA efficiency analysis. First case study is devoted to the derivation of closed form expression for maximal efficiency of PA for current-voltage pair of nonnegative cosine waveforms up to second harmonic and prescribed second harmonic resistive termination (Section 7.1). Section 7.2 provides an algorithm for calculation of efficiency of PA, providing that current is nonnegative cosine waveform with maximal amplitude of fundamental harmonic and voltage is nonnegative waveform with at least one zero and $b_{1}=0$. Closed form expression for maximal efficiency of PA for given second harmonic impedance, providing that voltage is nonnegative waveform up to second harmonic in general form and current is prescribed nonnegative cosine waveform, is presented in Section 7.3.

7.1. Current and Voltage Pair of Nonnegative Cosine Waveforms in PA Efficiency Analysis. In this subsection, we derive closed form expression for maximum efficiency of PA for given resistive second harmonic termination, providing that both current and voltage are nonnegative cosine waveforms. We also provide closed form expressions for coefficients of these waveforms. 
Let us consider generic PA circuit diagram, as shown in Figure 13. In what follows, we assume that voltage and current waveforms at the transistor output are

$$
\begin{gathered}
v(\theta)=1+a_{1 v} \cos \theta+a_{2 v} \cos 2 \theta, \\
i(\theta)=1+a_{1 i} \cos \theta+a_{2 i} \cos 2 \theta,
\end{gathered}
$$

where $\theta$ stands for $\omega t$. For convenience, we normalize the voltage and current waveforms such that supply voltage and dc current are $V_{\mathrm{dc}}=1$ and $I_{\mathrm{dc}}=1$, respectively. Consequently, current and voltage waveforms at the load are

$$
\begin{aligned}
& v_{L}(\theta)=a_{1 v} \cos \theta+a_{2 v} \cos 2 \theta, \\
& i_{L}(\theta)=-a_{1 i} \cos \theta-a_{2 i} \cos 2 \theta .
\end{aligned}
$$

In terms of coefficients $a_{1 v}, a_{2 v}, a_{1 i}$, and $a_{2 i}$, the load resistances at fundamental and second harmonic are $r_{1}=-a_{1 v} / a_{1 i}$ and $r_{2}=-a_{2 v} / a_{2 i}$, respectively, and PA efficiency is $\eta=$ $-a_{1 i} a_{1 v} / 2$.

Since PA efficiency is positive, it follows that $a_{1 i} a_{1 v}<0$. Furthermore, the output network is passive and therefore the relations between coefficients $a_{2 i}$ and $a_{2 v}$ can be expressed as $a_{2 v}=-r_{2} a_{2 i}$ where $r_{2} \geq 0$, or $a_{2 i}=-g_{2} a_{2 v}$ where $g_{2} \geq 0$. Consequently, $a_{2 i} a_{2 v} \leq 0$.

In what follows, we consider only the case $a_{1 i}>0$ and $a_{1 v}<0$, since discussion related to the case $a_{1 v}>0$ and $a_{1 i}<$ 0 remain the same, after interchanging roles of current and voltage.

Let us now assume that $a_{2 i} \geq 0$. Then, according to (122)(123), the following relation between $a_{1 i}$ and $a_{2 i}$ holds

$$
a_{1 i}= \begin{cases}1+a_{2 i} & \text { for } 0 \leq a_{2 i} \leq \frac{1}{3} \\ \sqrt{8 a_{2 i}\left(1-a_{2 i}\right)} & \text { for } \frac{1}{3} \leq a_{2 i} \leq 1\end{cases}
$$

Clearly, $a_{2 i} \geq 0$ and

$$
a_{2 v}=-r_{2} a_{2 i}
$$

imply $a_{2 v} \leq 0$. Therefore, from $a_{2 v} \leq 0$ and (121), it follows that $a_{1 v}=-1-a_{2 v}$ and $-1 \leq a_{2 v} \leq 0$, which, together with (127), lead to

$$
a_{1 v}=-1+r_{2} a_{2 i}
$$

and $r_{2} \leq 1 / a_{2 i}$. According to (128), increase of $r_{2}$ lead to decrease of $\left|a_{1 v}\right|$, which further lead to decrease of efficiency. Therefore, in the quest for maximum efficiency, it is enough to consider $r_{2} \in[0,1]$. According to (126) and (128), efficiency of PA can be expressed as an explicit function of $a_{2 i}$ and $r_{2}$,

$$
\eta= \begin{cases}\frac{1}{2}\left(1+a_{2 i}\right)\left(1-r_{2} a_{2 i}\right) & \text { for } 0 \leq a_{2 i} \leq \frac{1}{3} \\ \sqrt{2 a_{2 i}\left(1-a_{2 i}\right)}\left(1-r_{2} a_{2 i}\right) & \text { for } \frac{1}{3} \leq a_{2 i} \leq 1 .\end{cases}
$$

In order to find $a_{2 i}$ for which maximal efficiency is attained, first derivative of $\eta$ with respect to $a_{2 i}$ is set to zero. From

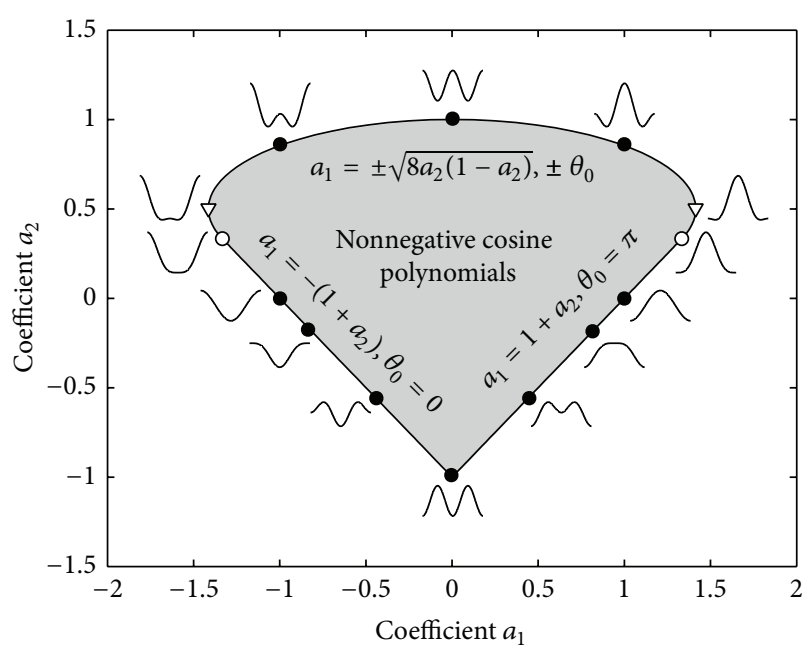

FIGURE 12: Parameter space of cosine polynomials.

$\partial \eta / \partial a_{2 i}=0$, we obtain coefficient $a_{2 i}$ as an explicit function of $r_{2}$ only:

$$
a_{2 i}= \begin{cases}\frac{1}{2} & \text { for } r_{2}=0, \\ \frac{3 r_{2}+2-\sqrt{9 r_{2}^{2}-4 r_{2}+4}}{8 r_{2}} & \text { for } 0<r_{2} \leq \frac{3}{5}, \\ \frac{1-r_{2}}{2 r_{2}} & \text { for } \frac{3}{5} \leq r_{2} \leq 1 .\end{cases}
$$

Notice that $1 / 3 \leq a_{2 i} \leq 1 / 2$ when $0 \leq r_{2} \leq 3 / 5$ and $0 \leq a_{2 i} \leq$ $1 / 3$ when $3 / 5 \leq r_{2} \leq 1$. Insertion of (130) into (126)-(129) provides closed form expressions for maximal efficiency and corresponding coefficients of waveforms in terms of $r_{2}$ only.

Let us now assume that $a_{2 v} \geq 0$. Repeating all arguments of the previous consideration, the following series of interchanging $a_{1 i} \leftrightarrow-a_{1 v}, r_{2} \rightarrow g_{2}$, and $a_{2 i} \leftrightarrow a_{2 v}$ lead to analogous result.

Procedure for calculation of maximal efficiency of PA with cosine waveforms for given second harmonic resistive termination and coefficients of corresponding waveforms, taking into account that $a_{1 i}>0$ and $a_{2 i} \geq 0$, is provided in the following algorithm.

Algorithm 20. One has the following steps:

(i) choose $r_{2} \in[0,1]$;

(ii) calculate $a_{2 i}$ from (130);

(iii) calculate $a_{1 i}, a_{2 v}$, and $a_{1 v}$ from (126), (127), and (128), respectively;

(iv) calculate efficiency $\eta=-a_{1 i} a_{1 v} / 2$.

The related graph of maximal efficiency $\eta$ and corresponding coefficients $a_{1 v}, a_{2 v}, a_{1 i}$, and $a_{2 i}$ are shown in Figure 14.

As an example, for prescribed $r_{2}=0.2$, a pair of cosine waveforms (124) that provides maximum efficiency is shown in Figure 15. By applying step (ii) of Algorithm 20, we obtain $a_{2 i}=0.4458$ and then, according to step (iii), we obtain $a_{1 i}=$ 1.4059, $a_{2 v}=-0.0892$, and $a_{1 v}=-0.9108$. Corresponding 


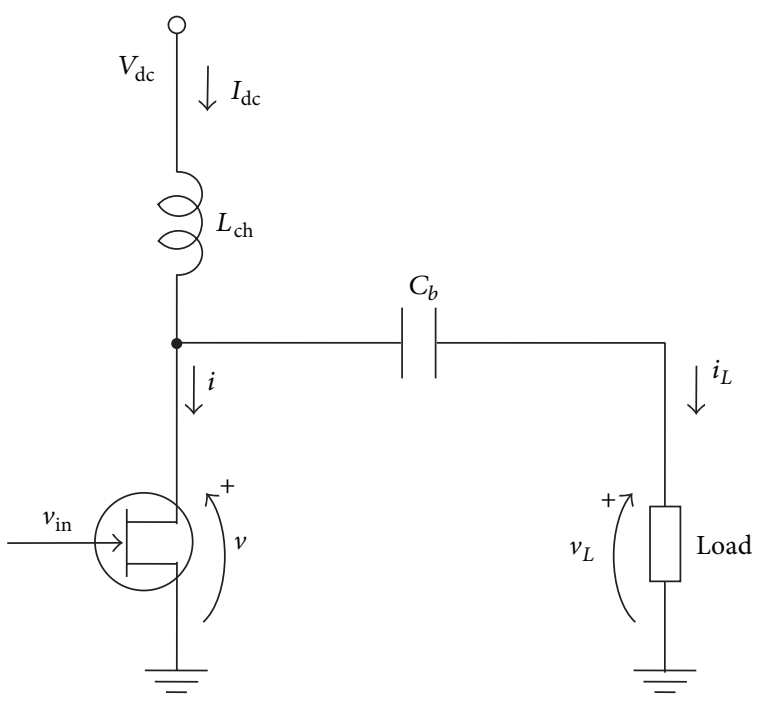

FIgURE 13: Generic PA circuit diagram.

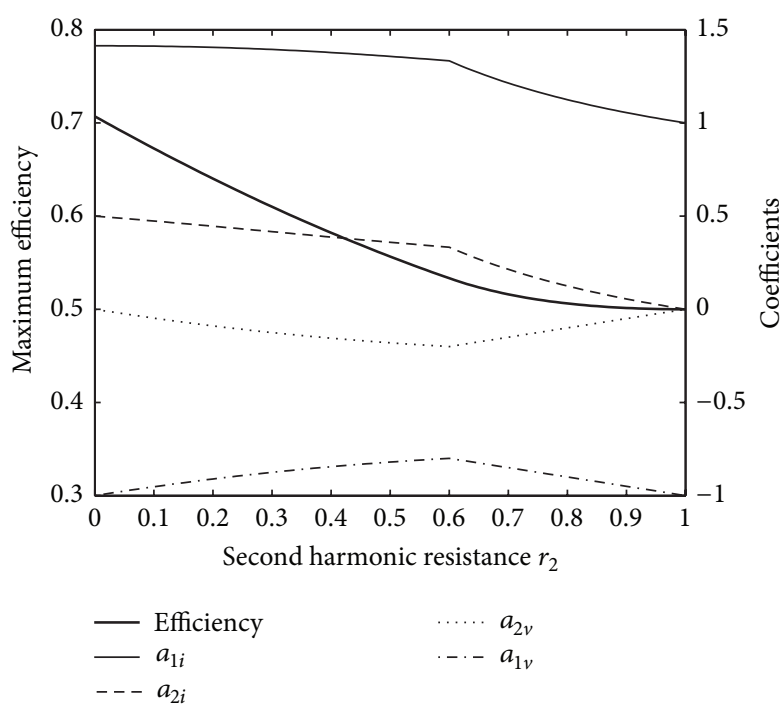

FIGURE 14: Maximal efficiency of PA and coefficients of corresponding cosine waveforms as function of second harmonic resistance $r_{2}$.

efficiency and load resistance at fundamental harmonic are $\eta=0.6403$ and $r_{1}=0.6479$, respectively.

The analytical results obtained in this subsection are in agreement with those obtained in [4] via numerical optimization process.

7.2. Nonnegative Waveforms with at Least One Zero and $b_{1}=$ 0 . Let us consider nonnegative waveforms of type (1) with $b_{1}=0$ and zero at instance $\theta_{0}$. In this subsection, we provide closed form expressions for the coefficients $a_{1}$ and $b_{2}$ of these waveforms in terms of coefficient $a_{2}$ and instance $\theta_{0}$.

The algorithm for calculation of efficiency of PA, providing that voltage is nonnegative waveform of type (1) with $b_{1}=0$ having at least one zero and current is nonnegative

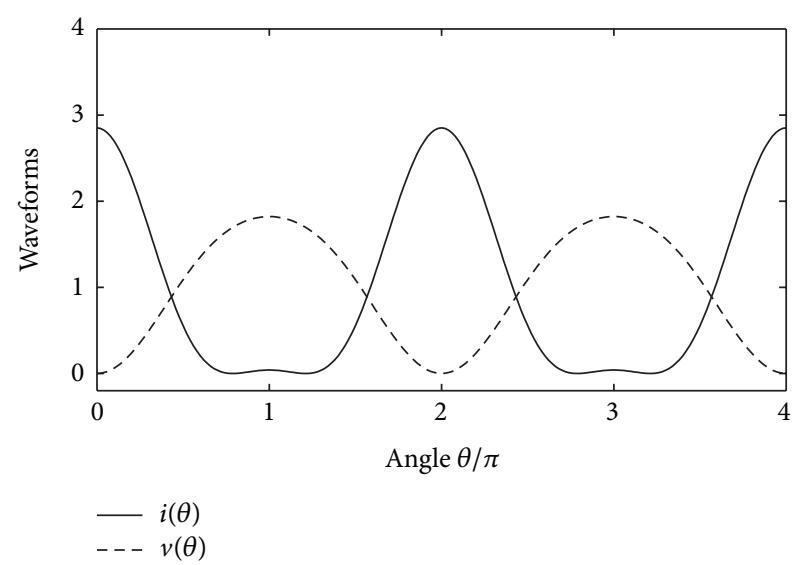

FIGURE 15: Pair of cosine waveforms (124) that provides maximum efficiency for $r_{2}=0.2$.

cosine waveform with maximal amplitude of fundamental harmonic, is presented.

As shown in Section 5.1, coefficients $a_{1}$ and $b_{1}$ of nonnegative waveforms with at least one zero can be expressed as a function of $\theta_{0}, a_{2}$, and $b_{2}$ (see (112)-(113)). Assumption $b_{1}=0$ together with (113) lead to

$$
b_{2}=\frac{1}{2} \tan \theta_{0}\left[3 a_{2}-1-\left(1-a_{2}\right) \tan ^{2} \theta_{0}\right] .
$$

Substitution of (131) into (112) yields

$$
a_{1}=-\cos \theta_{0}\left(1+\tan ^{2} \theta_{0}\right)\left[1+a_{2}-\left(1-a_{2}\right) \tan ^{2} \theta_{0}\right] .
$$

Furthermore, substitution of (131) into (102) and then insertion of resulting relations into $(\sin 2 \psi \cos \beta)^{2}+$ $(\sin 2 \psi \sin \beta)^{2}=\sin ^{2} 2 \psi \leq 1$ lead to the following inequality:

$$
\left[3 a_{2}-1-\left(1-a_{2}\right) \tan ^{2} \theta_{0}\right]\left[1+a_{2}-\left(1-a_{2}\right) \tan ^{2} \theta_{0}\right] \leq 0 .
$$

Because of $\left|a_{2}\right| \leq 1$, this inequality is equivalent to

$$
\max \left(0, \frac{3 a_{2}-1}{1-a_{2}}\right) \leq \tan ^{2} \theta_{0} \leq \frac{1+a_{2}}{1-a_{2}} .
$$

From (132) and (134), it is easy to see that

$$
\begin{array}{ll}
\theta_{0} \in\left(\frac{\pi}{2}, \frac{3 \pi}{2}\right) & \text { if } a_{1}>0, \\
\theta_{0} \in\left(\frac{-\pi}{2}, \frac{\pi}{2}\right) & \text { if } a_{1}<0 .
\end{array}
$$

Notice that nonnegative cosine waveforms with at least one zero can be obtained from (131), (132), and (134) by setting $b_{2}$ to zero. According to (131) and (134), $b_{2}=0$ implies that $\tan \theta_{0}=0$ for $a_{2} \leq 1 / 3$ and $\tan ^{2} \theta_{0}=\left(3 a_{2}-1\right) /\left(1-a_{2}\right)$ for $1 / 3<a_{2} \leq 1$, which, together with (132), lead to (121)-(123).

In what follows, we consider the efficiency of PA (see Figure 13), providing that current waveform is nonnegative 
cosine waveform with maximal amplitude of fundamental harmonic and voltage waveform is nonnegative waveform with at least one zero and $b_{1}=0$ :

$$
\begin{aligned}
& i(\theta)=1+\sqrt{2} \cos \theta+\frac{1}{2} \cos 2 \theta, \\
& v(\theta)=1+a_{1 v} \cos \theta+a_{2 v} \cos 2 \theta+b_{2 v} \sin 2 \theta .
\end{aligned}
$$

Fundamental harmonic impedance is $z_{1}=-a_{1 v} / a_{1 i}=$ $-a_{1 v} / \sqrt{2}$ (purely real) and second harmonic impedance is $\underline{z}_{2}=-\left(a_{2 v}-j b_{2 v}\right) / a_{2 i}=-2\left(a_{2 v}-j b_{2 v}\right)$, where $a_{1 i}=\sqrt{2}$ and $a_{2 i}=1 / 2$ for current waveform (136). Since the load is passive, it follows that $z_{1}>0$ and $\operatorname{Re}\left\{\underline{z}_{2}\right\} \geq 0$ which further imply $a_{1 v}<0$ and $a_{2 v} \leq 0$, respectively.

A procedure for calculation of coefficients of voltage waveforms (137) and corresponding efficiency for the voltagecurrent pair (137)-(136), taking into account that $a_{1 v}<0$ and $a_{2 v} \leq 0$, is presented in the following algorithm.

Algorithm 21. One has the following steps:

(i) choose $-1<a_{2 v} \leq 0$; (ii) choose $\frac{\theta_{0 v} \quad \text { from }}{-\arctan \sqrt{\left(1+a_{2 v}\right) /\left(1-a_{2 v}\right)}} \leq$ the $\theta_{0 v}$ range
$\arctan \sqrt{\left(1+a_{2 v}\right) /\left(1-a_{2 v}\right)} ;$

(iii) calculate $b_{2 v}$ and $a_{1 v}$ from (131) and (132), respectively;

(iv) calculate efficiency $\eta=-a_{1 v} / \sqrt{2}$.

Efficiency as a function of normalized second harmonic impedance $\underline{z}_{2} / z_{1}$ is presented in Figure 16 .

As an example, for prescribed coefficient $a_{2 v}=-0.1$ of nonnegative voltage waveform of type (137), according to step (ii) of Algorithm 21, the range for $\theta_{0 v}$ is [ $\left.-0.7353,0.7353\right]$. Let us choose $\theta_{0 v}=0.2$ from this range. Then, according to steps (iii) and (iv), the remaining coefficients and efficiency are $b_{2 v}=-0.1363, a_{1 v}=-0.8722$, and $\eta=0.6167$. Fundamental harmonic impedance and second harmonic impedance are equal to $z_{1}=0.6167$ and $\underline{z}_{2}=0.2-j 0.2727$, respectively. The voltage waveform and the associated current waveform given by (136) are presented in Figure 17.

7.3. Maximal Efficiency of PA with Prescribed Cosine Current Waveform. Let us consider current-voltage pair, such that voltage is nonnegative waveform of type (1) and current waveform is prescribed nonnegative cosine waveform:

$$
\begin{aligned}
& v(\theta)=1+a_{1 v} \cos \theta+b_{1 v} \sin \theta+a_{2 v} \cos 2 \theta+b_{2 v} \sin 2 \theta \\
& i(\theta)=1+a_{1 i} \cos \theta+a_{2 i} \cos 2 \theta
\end{aligned}
$$

Load impedances at fundamental and second harmonic are $\underline{z}_{1}=-\left(a_{1 v}-j b_{1 v}\right) / a_{1 i}$ and $\underline{z}_{2}=-\left(a_{2 v}-j b_{2 v}\right) / a_{2 i}$, respectively. Since the load is passive, it follows that $\operatorname{Re}\left\{\underline{z}_{1}\right\} \geq 0$ and $\operatorname{Re}\left\{\underline{z}_{2}\right\} \geq 0$ which further imply $a_{1 i} a_{1 v}<0$ and $a_{2 i} a_{2 v} \leq 0$. Efficiency of PA can be calculated as $\eta=-a_{1 i} a_{1 v} / 2$.

In this subsection, we consider the problem of finding maximal efficiency of PA with waveform pair (138)-(139) for given second harmonic impedance, providing that current waveform (139) is prescribed. It is easy to see that this problem can be reduced to the problem of finding voltage waveform with maximal coefficient $\left|a_{1 v}\right|$, for prescribed coefficients of second harmonic, which is already solved in Section 4 (see Proposition 9 and Remark 11).

In what follows, we will present an algorithm for calculation of maximal efficiency presuming that $a_{1 i}>0$ and $a_{2 i} \geq 0$. For other possible choices of signs of coefficients $a_{1 i}$ and $a_{2 i}$, only step (iii) of this algorithm should be modified in accordance to Remark 11.

Algorithm 22. One has the following steps:

(i) choose $\underline{z}_{2}=r_{2}+j x_{2}$ such that $\left|\underline{z}_{2}\right| \leq 1 /\left|a_{2 i}\right|$;

(ii) calculate $a_{2 v}-j b_{2 v}=-\underline{z}_{2} a_{2 i}$ and $\left|A_{2 v}\right|=\left|\underline{z}_{2} a_{2 i}\right|$;

(iii) if $2\left|A_{2 v}\right| \leq 1-a_{2 v}$, then calculate $a_{1 v}=-1-a_{2 v}$ and $b_{1 v}=-2 b_{2 v}$;

else, calculate $a_{1 v}=-2 \sqrt{\left(1-\left|A_{2 v}\right|\right)\left(\left|A_{2 v}\right|+a_{2 v}\right)}$ and $b_{1 v}=$ $-2 \operatorname{sgn}\left(b_{2 v}\right) \sqrt{\left(1-\left|A_{2 v}\right|\right)\left(\left|A_{2 v}\right|-a_{2 v}\right)}$;

(iv) calculate efficiency $\eta=-a_{1 i} a_{1 v} / 2$;

(v) calculate $\underline{z}_{1}=-\left(a_{1 v}-j b_{1 v}\right) / a_{1 i}$ and $\underline{z}_{2 n}=\underline{z}_{2} / \operatorname{Re}\left\{\underline{z}_{1}\right\}$.

Notice that step (iii) in above algorithm lead to the closed form expression for maximal efficiency of PA for given second harmonic impedance $\underline{z}_{2}=r_{2}+j x_{2}$,

$$
\eta=\left\{\begin{array}{l}
\frac{1}{2} a_{1 i}\left(1-r_{2} a_{2 i}\right) \\
\quad \text { for } 2\left|\underline{z}_{2} a_{2 i}\right| \leq 1+r_{2} a_{2 i}, \\
a_{1 i} \sqrt{\left(1-\left|\underline{z}_{2} a_{2 i}\right|\right)\left(\left|\underline{z}_{2} a_{2 i}\right|-r_{2} a_{2 i}\right)} \\
\quad \text { for } 2\left|\underline{z}_{2} a_{2 i}\right|>1+r_{2} a_{2 i},
\end{array}\right.
$$

providing that current waveform is nonnegative cosine waveform with coefficients $a_{1 i}>0$ and $a_{2 i} \geq 0$ and voltage waveform has the form (138).

For the waveform pair (136) and (138), where current waveform (136) is nonnegative cosine waveform of type (139) with maximal coefficient $a_{1 i}$, maximal efficiency of PA as a function of normalized second harmonic impedance $\underline{z}_{2 n}=$ $\underline{z}_{2} / \operatorname{Re}\left\{\underline{z}_{1}\right\}$ is presented in Figure 18 .

Notice that Figures 18 and 16 show that the highest efficiency (about 0.7 ) is attained when second harmonic load is close to the purely reactive (see the edges of Smith charts presented in Figures 18 and 16). This efficiency of 0.7 is close to the theoretical upper bound $\sqrt{2} / 2 \approx 0.7071$ for efficiency when both current and voltage are nonnegative waveforms up to second harmonic [4].

Let us consider two examples with current waveform (136) and prescribed second harmonic impedance, one related to the case $2\left|A_{2 v}\right| \leq 1-a_{2 v}$ and the other covering the case $2\left|A_{2 v}\right|>1-a_{2 v}$ (see step (iii) of the above algorithm). Thus, $\underline{z}_{2}=0.1-j 0.5$ and current waveform (136) imply that $2\left|A_{2 v}\right| \leq 1-a_{2 v}$. Then, according to the above algorithm, the coefficients of voltage waveform of type (138) which provide maximal efficiency are $a_{2 v}=-0.05, b_{2 v}=-0.25, a_{1 v}=-0.95$, and $b_{1 v}=0.5$ (see Figure 19). Corresponding efficiency and normalized second harmonic impedance are $\eta=0.6718$ and $\underline{z}_{2 n}=0.1489-j 0.7443$. On the other hand, $\underline{z}_{2}=0.1-j 1.1$ and current waveform (136) imply that $2\left|A_{2 v}\right|>1-a_{2 v}$. Then, 


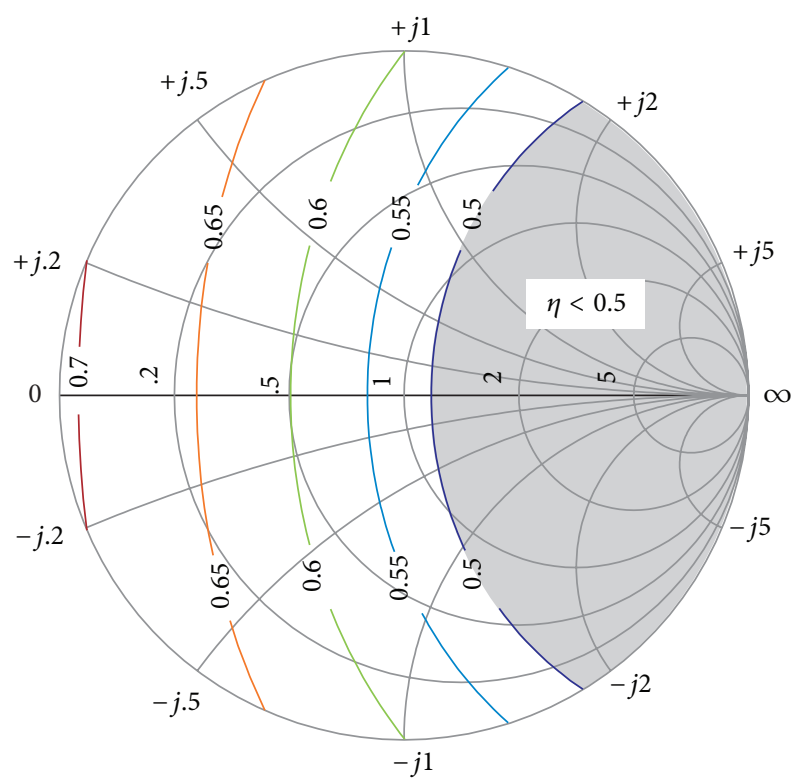

FIGURE 16: Efficiency contours of PA with waveform pair (136)-(137) as a function of normalized second harmonic impedance.

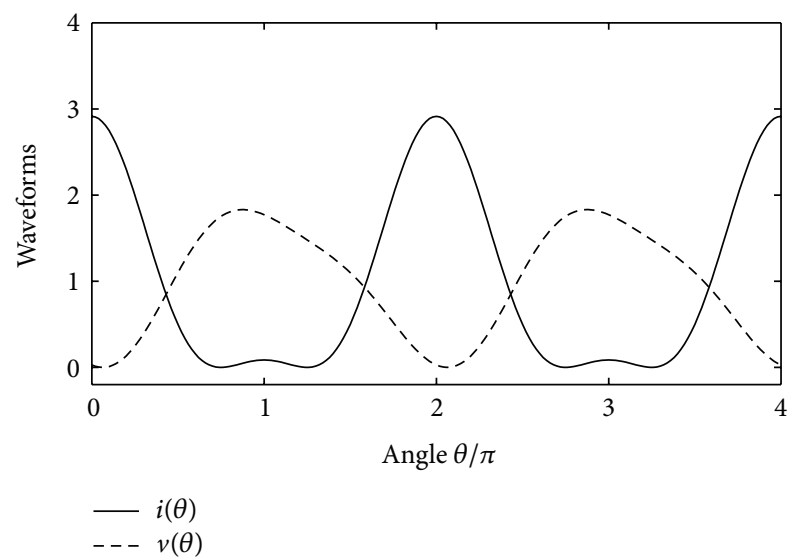

FIGURE 17: Current waveform (136) and voltage waveform (137) with $a_{2 v}=-0.1$ and $\theta_{0 v}=0.2$.

according to the above algorithm, the coefficients of voltage waveform of type (138) which provide maximal efficiency are $a_{2 v}=-0.05, b_{2 v}=-0.55, a_{1 v}=-0.9484$, and $b_{1 v}=1.0386$ (see Figure 20). Efficiency and normalized second harmonic impedance are $\eta=0.6706$ and $\underline{z}_{2 n}=0.1491-j 1.6402$.

\section{Conclusion}

In this paper, we provide general characterisation of nonnegative waveforms up to second harmonic in terms of four independent parameters (Proposition 1). Four important subclasses of the class of nonnegative waveforms are also fully characterised: nonnegative waveforms with maximal amplitude of fundamental harmonic for prescribed amplitude of

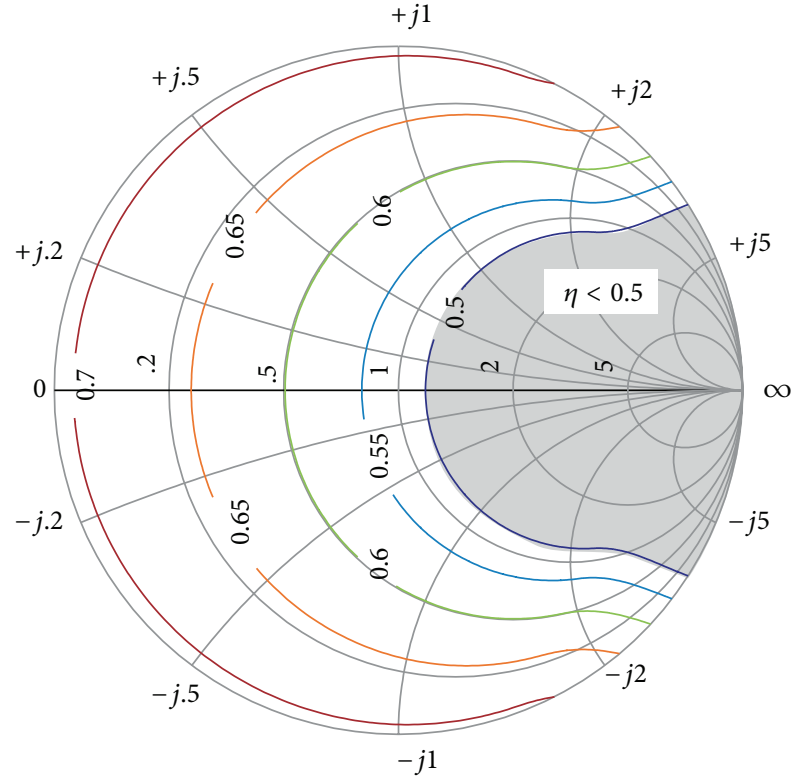

FIGURE 18: Contours of maximal efficiency of PA with waveform pair (136) and (138) as a function of normalized second harmonic impedance $\underline{z}_{2 n}=\underline{z}_{2} / \operatorname{Re}\left\{\underline{z}_{1}\right\}$.

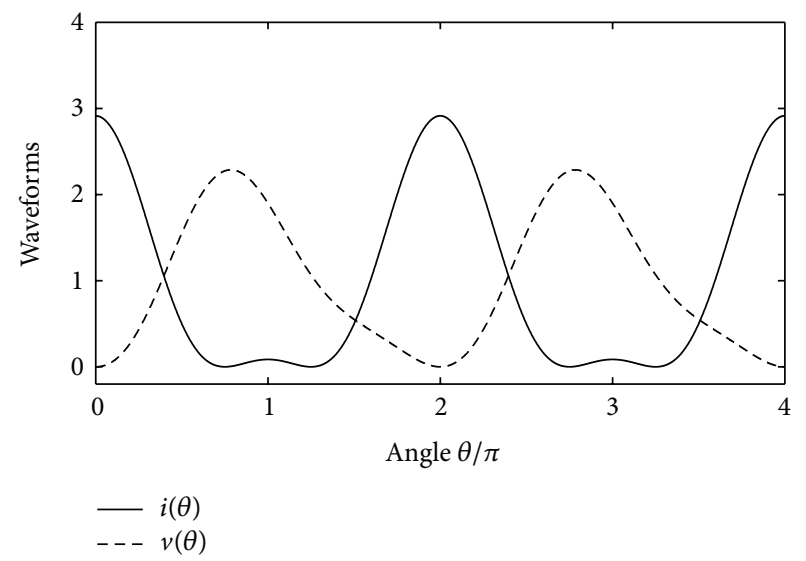

FIGURE 19: Current waveform (136) and nonnegative voltage waveform of type (138) that in pair with (136) provides maximal efficiency for $\underline{z}_{2}=0.1-j 0.5$.

second harmonic (Proposition 4), nonnegative waveforms with maximal coefficient of cosine part of fundamental harmonic for prescribed coefficients of second harmonic (Proposition 9), nonnegative waveforms with at least one zero (Proposition 12), and nonnegative cosine waveforms with at least one zero (Proposition 18). An algorithm (Algorithm 17) to facilitate calculation of coefficients $a_{1}$ and $b_{1}$ of nonnegative waveforms with at least one zero for prescribed coefficients $a_{2}$ and $b_{2}$ is also provided. Three case studies related to the efficiency of PA with nonnegative waveforms up to second harmonic, along with associated algorithms (Algorithms 2022), are considered in detail. 


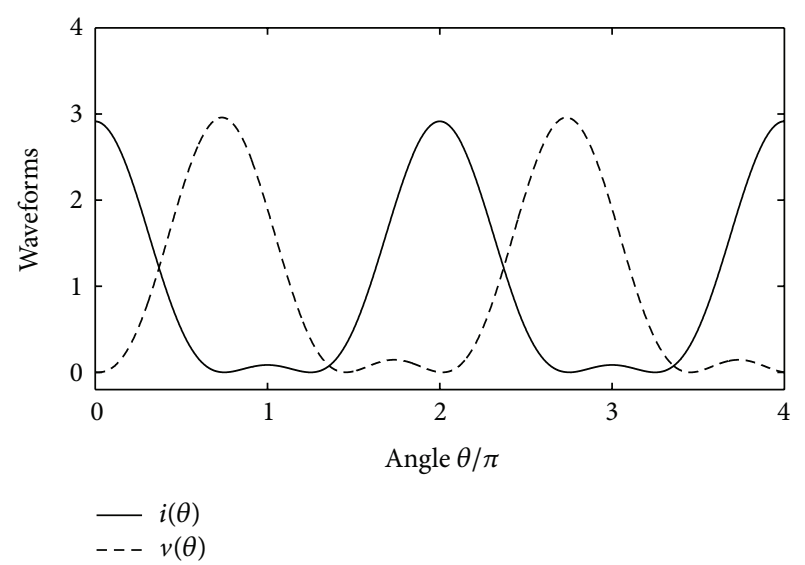

FIGURE 20: Current waveform (136) and nonnegative voltage waveform of type (138) that in pair with (136) provides maximal efficiency for $\underline{z}_{2}=0.1-j 1.1$.

\section{Conflict of Interests}

The authors declare that there is no conflict of interests regarding the publication of this paper.

\section{Acknowledgment}

This work is supported by Serbian Ministry of Education, Science and Technology Development as a part of the Project TP32016.

\section{References}

[1] F. H. Raab, "Class-E, class-C, and class-F power amplifiers based upon a finite number of harmonics," IEEE Transactions on Microwave Theory and Techniques, vol. 49, no. 8, pp. 1462-1468, 2001.

[2] J. D. Rhodes, "Output universality in maximum efficiency linear power amplifiers," International Journal of Circuit Theory and Applications, vol. 31, no. 4, pp. 385-405, 2003.

[3] A. Juhas and L. A. Novak, "Comments on 'Class-E, class$\mathrm{C}$, and class $\mathrm{F}$ power amplifier based upon a finite number of harmonics"' IEEE Transactions on Microwave Theory and Techniques, vol. 57, no. 6, pp. 1623-1625, 2009.

[4] M. Roberg and Z. Popović, "Analysis of high-efficiency power amplifiers with arbitrary output harmonic terminations," IEEE Transactions on Microwave Theory and Techniques, vol. 59, no. 8, pp. 2037-2048, 2011.

[5] A. Juhas and L. A. Novak, "Maximally flat waveforms with finite number of harmonics in class- $F$ power amplifiers," Mathematical Problems in Engineering, vol. 2013, Article ID 169590, 9 pages, 2013.

[6] T. Canning, P. J. Tasker, and S. C. Cripps, "Continuous mode power amplifier design using harmonic clipping contours: theory and practice," IEEE Transactions on Microwave Theory and Techniques, vol. 62, no. 1, pp. 100-110, 2014.

[7] A. Grebennikov, N. O. Sokal, and M. J. Franco, Switchmode RF Power Amplifiers, Elsevier/Academic Press, San Diego, Calif, USA, 2nd edition, 2012.
[8] L. Fejer, "Über trigonometrische polynome," Journal für die Reine und Angewandte Mathematik, vol. 1916, no. 146, pp. 5382, 1916 (German).

[9] H. L. Montgomery and U. M. A. Vorhauer, "Biased trigonometric polynomials," The American Mathematical Monthly, vol. 114, no. 9, pp. 804-809, 2007.

[10] K. Mimis, K. A. Morris, S. Bensmida, and J. P. McGeehan, "Multichannel and wideband power amplifier design methodology for $4 \mathrm{G}$ communication systems based on hybrid classJ operation," IEEE Transactions on Microwave Theory and Techniques, vol. 60, no. 8, pp. 2562-2570, 2012.

[11] S. Rezaei, L. Belostotski, F. M. Ghannouchi, and P. Aflaki, "Integrated design of a class-J power amplifier," IEEE Transactions on Microwave Theory and Techniques, vol. 61, no. 4, pp. 1639-1648, 2013.

[12] J. H. Kim, S. J. Lee, B. H. Park, S. H. Jang, J. H. Jung, and C. S. Park, "Analysis of high-efficiency power amplifier using second harmonic manipulation: inverse class-F/J amplifiers," IEEE Transactions on Microwave Theory and Techniques, vol. 59, no. 8, pp. 2024-2036, 2011.

[13] A. Dani, M. Roberg, and Z. Popovic, "PA efficiency and linearity enhancement using external harmonic injection," IEEE Transactions on Microwave Theory and Techniques, vol. 60, no. 12, pp. 4097-4106, 2012.

[14] G. V. Milovanović, D. S. Mitrinović, and Th. M. Rassias, Topics in Polynomials: Extremal Problems, Inequalities, Zeros, World Scientific, Singapore, 1994. 


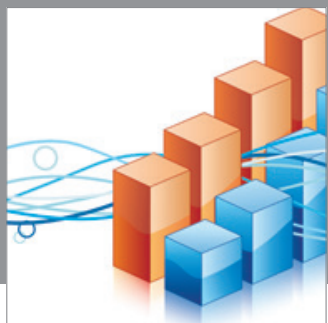

Advances in

Operations Research

mansans

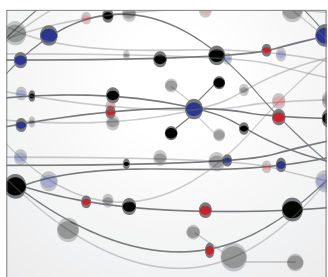

The Scientific World Journal
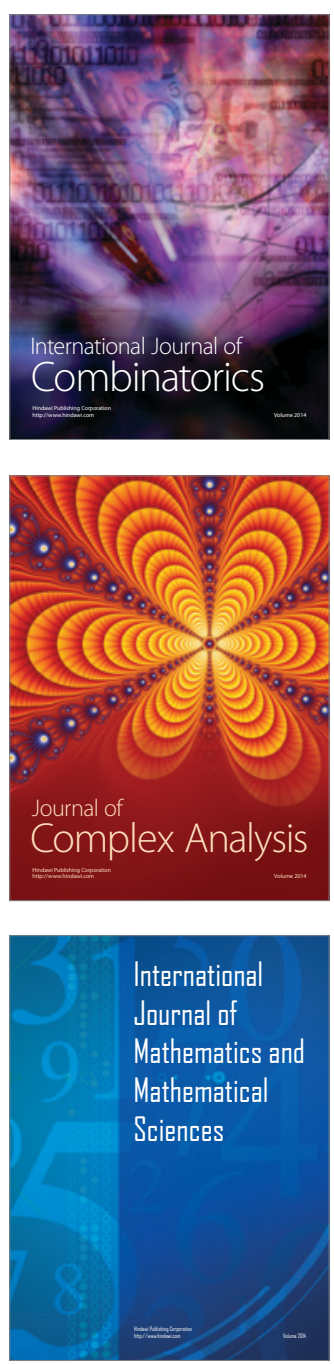
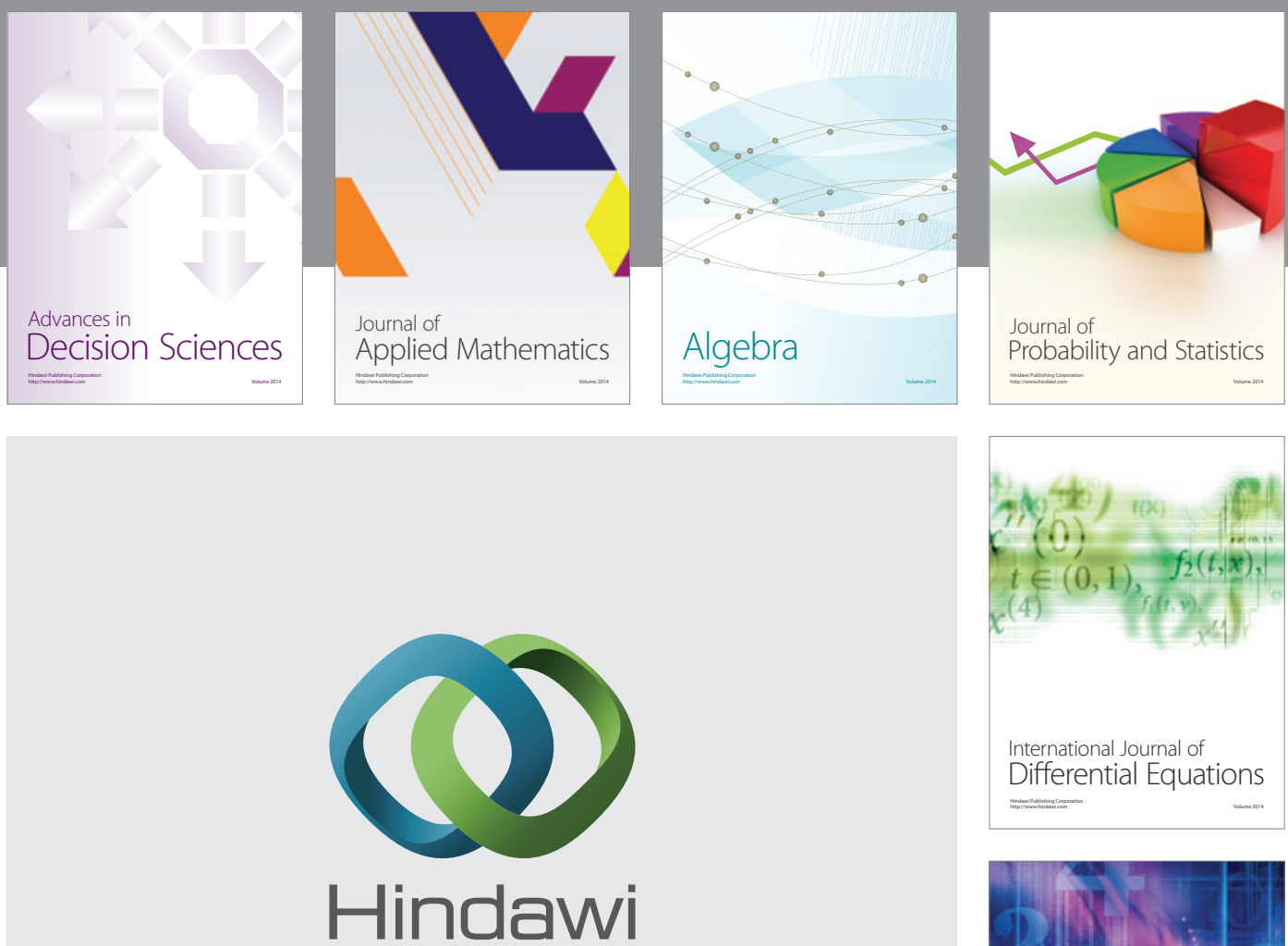

Submit your manuscripts at http://www.hindawi.com
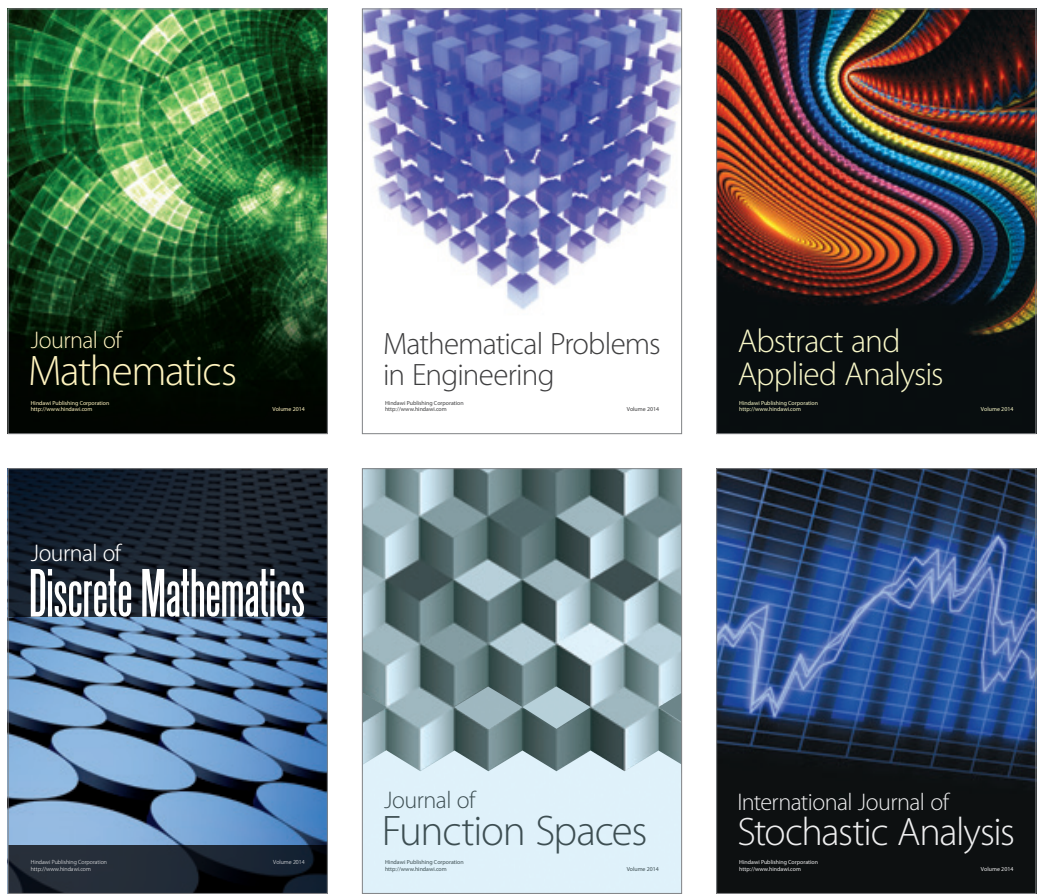

Journal of

Function Spaces

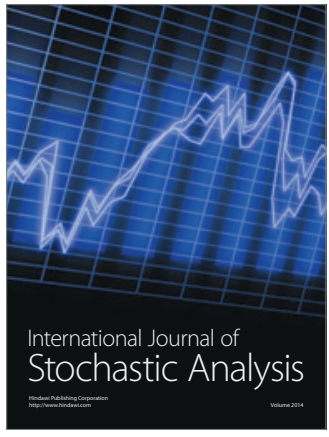

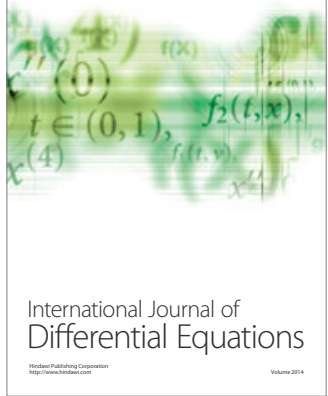
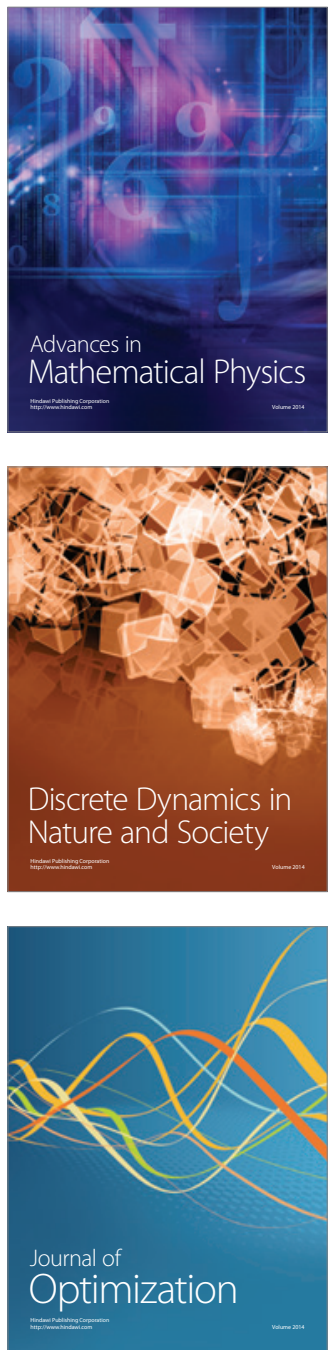